\title{
$\alpha$-Tocopherol Foliar Spray and Translocation Mediates Growth, Photosynthetic Pigments, Nutrient Uptake, and Oxidative Defense in Maize (Zea mays L.) under Drought Stress
}

\author{
Qasim Ali ${ }^{1, *}$, Muhammad Tariq Javed ${ }^{1}$, Muhammad Zulqurnain Haider ${ }^{1}{ }^{\mathbb{D}}$, Noman Habib ${ }^{1}$, \\ Muhammad Rizwan ${ }^{2}$ (D), Rashida Perveen ${ }^{3}$, Shafaqat Ali ${ }^{2,4}$,*(D), Mohammed Nasser Alyemeni ${ }^{5}$, \\ Hamed A. El-Serehy ${ }^{6}$ and Fahad A. Al-Misned ${ }^{6}$ \\ 1 Department of Botany, Government College University, New Campus, Jhang Road, \\ Faisalabad 38000, Pakistan; mtariqjaved@gcuf.edu.pk (M.T.J.); dr_mzhaider@yahoo.com (M.Z.H.); \\ nomi4442003@yahoo.com (N.H.) \\ 2 Department of Environmental Sciences and Engineering, Government College University, \\ Allama Iqbal Road, Faisalabad 38000, Pakistan; mrazi1532@yahoo.com \\ 3 Department of Physics, University of Agriculture, Faisalabad 38040, Pakistan; 2007ag942@uaf.edu.pk \\ 4 Department of Biological Sciences and Technology, China Medical University, Taichung 40402, Taiwan \\ 5 Botany and Microbiology Department, College of Science, King Saud University, Riyadh 11451, Saudi Arabia; \\ mnyemeni@ksu.edu.sa \\ 6 Department of Zoology, College of Science, King Saud University, Riyadh 11451, Saudi Arabia; \\ helserehy@ksu.edu.sa (H.A.E.-S.); almisned@ksu.edu.sa (F.A.A.-M.) \\ * Correspondence: drqasimali@gcuf.edu.pk (Q.A.); shafaqataligill@gcuf.edu.pk or \\ shafaqataligill@yahoo.com (S.A.)
}

Received: 21 June 2020; Accepted: 10 August 2020; Published: 21 August 2020

\begin{abstract}
A pot experiment was conducted to assess the induction of drought tolerance in maize by foliar-applied $\alpha$-tocopherol at early growth stage. Experiment was comprised two maize cultivars (Agaiti-2002 and EV-1098), two water stress levels (70\% and 100\% field capacity), and two $\alpha$-tocopherol levels $(0 \mathrm{mmol}$ and $50 \mathrm{mmol})$ as foliar spray. Experiment was arranged in a completely randomized design in factorial arrangement with three replications of each treatment. $\alpha$-tocopherol was applied foliary at the early vegetative stage. Water stress reduced the growth of maize plants with an increase in lipid peroxidation in both maize cultivars. Contents of non-enzymatic antioxidants and activities of antioxidant enzymes increased in studied plant parts under drought, while the nutrient uptake was decreased. Foliary-applied $\alpha$-tocopherol improved the growth of both maize cultivars, associated with improvements in photosynthetic pigment, water relations, antioxidative mechanism, and better nutrient acquisition in root and shoot along with tocopherol contents and a decrease in lipid peroxidation. Furthermore, the increase of tocopherol levels in roots after $\alpha$-Toc foliar application confers its basipetal translocation. In conclusion, the findings confer the role of foliar-applied $\alpha$-tocopherol in the induction of drought tolerance of maize associated with tissue specific improvements in antioxidative defense mechanism through its translocation.
\end{abstract}

Keywords: $\alpha$-Tocopherol; antioxidants; drought; nutrient dynamics; tissue specific response

\section{Introduction}

Among different environmental adversities, water shortage is of major focus, which has hampered the production of global agricultural systems [1,2]. At a global level, about $45 \%$ of all land is prevailed by drought [3]. On the other hand, an estimated increase in the world population will be about 
2.5 billion in the next 25 years, which will exert huge pressure on agriculture to fulfill world food demand and on the available freshwater resources. From the last two decades, Pakistan has also faced the problem of agricultural productivity to fulfill the food demand of the sixth largest population in the world. With an agriculture-based economy, Pakistan is predominantly categorized as arid country lying within the geographic coordinates of $23.38^{\circ}-30.25^{\circ} \mathrm{N}$ latitude and $61.78^{\circ}-74.30^{\circ} \mathrm{E}$ longitude, with a total land area of $796,096 \mathrm{~km}^{2}$ [4]. The interannual rainfall variability makes the arid region (covering 75\% land area of Pakistan) more susceptible to drought risks. Approximately 34.15 Mha of land area is in agriculture use, and uncultivated land is 23.60 Mha. About $25 \%$ of the cultivated land is rainfed, which plays a vital role in the country's economy [5]. Due to the major contribution of the agriculture sector in Pakistan's economy, Pakistan is more susceptible to drought risks [6]. In recent decades, unexpected and rapid changes in climate have severely affected socioeconomic and environmental conditions in Pakistan [6]. The major cause of drought stress is a decrease in soil water contents in combination with evaporation due to over-changing atmospheric conditions [7]. Shortage of water induces drastic changes in plants' physio-biochemical and molecular properties that ultimately affects all growth stages of a plant's life cycle, including the final yield [8,9]. At present (and in the near future), the maintenance of crop productivity for a large population under limited water supply is a challenge for the researchers working in the agriculture sector.

To survive under water deficit conditions, plants have manipulated metabolic defensive systems/mechanisms, which are species- and genotype-specific [10-12]. Disturbance in plant water status is the important effect of water shortage that triggers various other metabolic processes to survive under water stress $[10,11,13,14]$. It results in reduced growth and final grain yield due to perturbations in photosynthesis by disturbances in the biosynthesis of photosynthetic pigments and impaired nutrient uptake $[15,16]$. Water deficit conditions cause sub-optimal plant photosynthetic efficiency due to limited $\mathrm{CO}_{2}$ diffusion into the leaves due to less stomatal opening or reduced Rubisco activity $[17,18]$. To cope with a stressful environment, the plant mineral uptake mechanism plays a significant role in improving resistance $[19,20]$. Generally, under water deficit conditions, mineral uptake and transport reduces due to a decrease in the nutrient diffusion rate [16,21]. Among different nutrients, potassium $\left(\mathrm{K}^{+}\right)$, nitrogen $(\mathrm{N})$, calcium $\left(\mathrm{Ca}^{2+}\right)$, phosphorus $(\mathrm{P})$, and magnesium $\left(\mathrm{Mg}^{2+}\right)$ have prime importance due to their vital functions in plant physio-biochemical processes $[14,20,22]$.

The stress tolerance in crop plants that results in better yield is growth-stage and species-specific [23,24]. The seedling stage is of prime importance in potentially contributing to better seed yield. Uniform crop stand leads to better yield, which depends on better seedling growth $[25,26]$. Furthermore, it was found that at early seedling stages, crop cultivars with better antioxidative potential are more drought tolerant than cultivars with less antioxidative activity [27] because the disturbances in different physiological mechanisms results in another secondary stress (oxidative stress) by excessive production of reactive oxygen species (ROS).

Stress-induced oxidative stress due to production of $\operatorname{ROS}\left(\mathrm{O}_{2}^{-}, \mathrm{H}_{2} \mathrm{O}_{2}, \mathrm{OH}^{-}\right.$, and $\left.\mathrm{O}^{*}\right)$ is a common phenomenon in all organisms [28]. Over-production of ROS damages membrane lipids [28], thereby increasing malondialdehyde (MDA) accumulation due to limited activity of antioxidative defense mechanisms [29]. Under stressful environments, the levels of MDA are parallel with antioxidant enzyme activities, which are the indices to assess the status of the extent of damage due to the overproduction of ROS [30]. Other than the levels of antioxidant enzyme to counteract ROS damage, plants also have non-enzymatic antioxidative defense mechanisms such as the production of ascorbic acid, phenolic acid, carotenoids, tocopherols, etc. [31]. Furthermore, it is well known that the antioxidative defensive phenomenon is inter-species, cultivar, and growth-stage-specific. However, most of the higher yielding genotypes are not drought tolerant when considering stress tolerance mechanisms [32].

Furthermore, some high-yield crop cultivars are deficit with regard to such anti-stress mechanisms $[14,28,33]$. For the induction of drought tolerance, different approaches have been adopted, including the exogenous application of secondary growth metabolic compounds [34-37]. Exogenous 
application such as the foliar spray of different secondary metabolites of which the plant is in deficit is considered as an effective means among others for stress tolerance induction [38,39]. It is well known that foliar application of such compounds is translocatable to different plant parts. Furthermore, after their translocation to different plant parts, they play a potential role in the induction of drought tolerance. Along with modulating metabolic activities, plants also control their own metabolisms [34,40]. Among different secondary metabolic compounds, the tocopherols are lipophilic in nature and scavenge ROS, with the ability to recycle themselves and, as a result, reduce lipid peroxidation. Tocopherols belong to a family of eight members including $\alpha, \beta, \gamma$, and $\delta$ tocopherols, along with their respective precursors (tocotrienols) that have high antioxidative activity and protect plants from stress through different metabolic processes [41]. Among these, $\alpha$-Toc is largely known as vitamin E, with large antioxidant potential in comparison with other family members, but the production of $\alpha$-Toc to reduce oxidative damage is cultivar-specific [42]. However, $\alpha$-Toc exogenous application was found to be helpful for stress tolerance induction. For example, in wheat, exogenous application of $\alpha$-Toc improved salt-stress tolerance [43]. In flax, genotypes foliar-applied tocopherol significantly improved salt stress tolerance [44]. Most of the studies presented are regarding salt tolerance induction and the application of $\alpha$-Toc on adult-stage plants, and there is a lack of knowledge regarding its exogenous use at other growth stages. However, the discovery of the proper plant stage for better drought-stress induction through exogenous use of this compound is of prime importance [45].

Furthermore, there are missing gaps in understanding the proper physiological mechanism for the induction of stress tolerance at different growth stages by the exogenous use of organic compounds like that of $\alpha-T o c$, also considering its translocation to specific plant parts. Therefore, the current work was aimed to quantify to which extent the foliar applied $\alpha$-Toc could modulate growth in water-stressed maize plants and when it should be applied in the early growth stage. The goal of the study was to draw parallels among tissue-specific alterations in endogenous tocopherol levels, antioxidative defense mechanisms, and nutrient mobility patterns after $\alpha$-Toc foliar application in maize plants grown in a drought-stressed rhizosphere. The research outcomes are helpful for optimizing strategies for growing maize with limited irrigation and in semi-arid and arid regions for better growth and production.

Maize (Zea mays L.) is the third most commonly produced cereal, after wheat and rice. It has a potential to grow in a wide range of environmental conditions and has gained great economic priority due to its potential nutritional quality all over the world, including in Pakistan [46]. In Pakistan, 1.016 million hectares are under maize cultivation, and $35 \%$ of the total cultivated area is rainfed, which is now facing problems in getting better production under dry environmental spells; this situation has further become more severe due to the present change in environmental conditions. Maize kernels are not only good and cheap source of carbohydrates but are also a rich source of carotenoids, proteins, and edible oil. However, due to changes in rainfall patterns along with the shortage of fresh water for irrigation, its production is under threat, along with that of other crops.

\section{Materials and Methods}

The present experiment was arranged in the research area of the Department of Botany, Government College University Faisalabad, Pakistan, (latitude $30^{\circ} 30 \mathrm{~N}$, longitude $73^{\circ} 10 \mathrm{E}$, and altitude $213 \mathrm{~m}$ ) under natural environmental conditions during August-September 2018. To avoid disturbances due to rain, the experimental area was covered with a polyethylene sheet. The design of the experiment was completely randomized in factorial arrangement, with three replications of each treatment. The experiment consisted of two drought levels (control and 70\% field capacity), two highly yielding maize genotypes (EV-1098 and Agaiti-2002), and two levels of $\alpha$-Toc $(0 \mathrm{mmol}$ and $50 \mathrm{mmol})$ in solution form applied as foliar spray with three replications of each treatment. The $70 \%$ field capacity used in the present study was selected following some earlier studies $[47,48]$. These two maize cultivars selected for study are used frequently in breeding programs to produce high-yielding hybrid genotypes. The experimental unit was comprised a total 24 equal-size plastic pots $(28 \mathrm{~cm} \times 30 \mathrm{~cm})$, each filled with $10 \mathrm{~kg}$ soil. The soil was fully irrigated with canal water before seed sowing. When the soil 
was at field capacity, seeds of both maize genotypes were hand sown. Before sowing, the soil was prepared well by hand digging. The seeds of both maize genotypes were purchased from Maize and Millet Research Institute, Yousafwala Sahiwal, Pakistan. Ten healthy seeds were sown in each pot. After five days of the completion of seed germination, five seedlings per pot were maintained by thinning. The water stress treatment was started just after the thinning of the seedlings by controlling the irrigation of half of the pots at $70 \%$ field capacity, and the other half of the pots were treated as control plants and irrigated to maintain 100\% field capacity. Average mean daily length was $13 / 11 \mathrm{~h}$, mean minimum and maximum day/night temperatures were $38 \pm 3 / 30 \pm 3{ }^{\circ} \mathrm{C}$ and $25 \pm 2.5 / 20 \pm 2.5^{\circ} \mathrm{C}$, respectively, the mean relative humidity during whole experiment (at daytime) was 50\%. During the whole experimental period the averaged photosynthetically available radiation (PAR) measured at noon was varied from $794 \mu \mathrm{molm}^{-2} \mathrm{~s}^{-1}$ to $1154 \mu \mathrm{molm}^{-2} \mathrm{~s}^{-1}$. Soil moisture content was maintained on daily basis and using a tensiometer, (Irrometer, Model RT-12 inch Riverside, CA, USA). Ten days after thinning, the seedlings were supplied exogenously as foliar spray with $0 \mathrm{mmol}$ and $50 \mathrm{mmol}$ solution of $\alpha$-toc. Foliar spray of $\alpha$-Toc solution was done in evening before sunset for the maximum absorption of the solution in leaf. The spray of $\alpha$-Toc solution was made only once during the whole experimental period. An aliquot of $50 \mathrm{~mL}$ solution of each of $\alpha$-Toc level was applied manually per replicate as foliar spray that costs only \$0.015 USD for six plants and \$65 USD per acre. The solution was prepared by dissolving the required measured quantity in minimal amount of ethanol, and then the final volume was maintained with distilled water. The 0 mmol treatment without $\alpha$-Toc was considered as control treatment. Before foliar spray, $0.1 \%$ of Tween- 20 was added as the surfactant to the finally prepared solution for the maximum absorption of the solution. The data for varying attributes was calculated after 15 days of $\alpha$-Toc foliar spray. Fresh leaf material was taken in liquid nitrogen and stored at $-80^{\circ} \mathrm{C}$ for different biochemical studies.

\subsection{Soil Analysis}

The soil used was sandy loam with a saturation percentage of 47.5 , average $\mathrm{pH}$, and the ECe of the soil solution was $7.63 \mathrm{ds} . \mathrm{m}^{-1}$ and $0.045 \mathrm{ds} . \mathrm{m}^{-1}$, respectively, organic matter $(1.21 \%)$, with the available $\mathrm{P}\left(0.051 \mathrm{mg} \mathrm{kg}^{-1}\right), \mathrm{K}\left(30 \mathrm{mg} \mathrm{kg}^{-1}\right)$, and total $\mathrm{N}\left(6.1 \mathrm{mg} \mathrm{kg}^{-1}\right)$. The soil solution had soluble $\mathrm{CO}_{3}{ }^{2-}$ (traces), $\mathrm{HCO}_{3}{ }^{-}\left(5.01 \mathrm{meq} \mathrm{L}{ }^{-1}\right), \mathrm{Cl}^{-}\left(8.49 \mathrm{meq} \mathrm{L}^{-1}\right), \mathrm{SO}_{4}{ }^{-2}\left(2.01 \mathrm{meq} \mathrm{L}{ }^{-1}\right)$, Na $\left(3.01 \mathrm{meq} \mathrm{L}^{-1}\right)$, $\mathrm{Ca}^{2+}+\mathrm{Mg}^{2+}\left(13.91 \mathrm{meq} \mathrm{L}^{-1}\right)$, and SAR $\left(0.079\right.$ meq L $\left.^{-1}\right)$.

\subsection{Estimation of Different Growth Parameters}

Two plants per replicate were uprooted and washed with distilled water for the estimation of different growth attributes. After calculating root and shoot lengths, number of leaves, leaf area, and fresh masses of roots and shoots, the same plants was then oven-dried using an electric oven at $70^{\circ} \mathrm{C}$ for $48 \mathrm{~h}$, and their dry masses were calculated.

\subsection{Estimation of Leaf Photosynthetic Pigments}

For the estimation of leaf chlorophyll (Chl.) $a, b$, total Chl, and Chl $a / b$, we followed the method described by Arnon [49]. The content of carotenoids (Car) was estimated following Kirk and Allen [50]. The extraction of the pigments was done using $80 \%$ acetone. Briefly, fresh leaf material $(0.1 \mathrm{~g})$ was chopped and put in $10 \mathrm{~mL}$ acetone for overnight at $4{ }^{\circ} \mathrm{C}$ and the absorbance of the extract was read at 663,645 , and $480 \mathrm{~nm}$ using a spectrophotometer (Hitachi U-2001, Tokyo, Japan). The quantities were computed using the specific formulae:

$$
\begin{gathered}
\text { Chl. } a=[12.7(\mathrm{OD} 663)-2.69(\mathrm{OD} 645)] \times \mathrm{V} / 1000 \times \mathrm{W} \\
\text { Chl. } b=[22.9(\mathrm{OD} 645)-4.68(\mathrm{OD} 663)] \times \mathrm{V} / 1000 \times \mathrm{W} \\
\text { Total Chl. }=[20.2(\Delta \mathrm{A} 645)-8.02(\Delta \mathrm{A} 663)] \times \mathrm{v} / \mathrm{w} \times 1 / 1000
\end{gathered}
$$




$$
\begin{gathered}
\text { A carotenoid }(\mu \mathrm{g} / \mathrm{g} F W)=\Delta \mathrm{A} 480+(0.114 \times \Delta \mathrm{A} 663)-(0.638 \times \Delta \mathrm{A} 645) \\
\mathrm{Car}=\mathrm{A} \text { Car. } / \mathrm{Em} 100 \% \times 100 \\
\text { Emission }=\mathrm{Em} 100 \%=2500 \\
\Delta \mathrm{A}=\text { absorbance at respective wavelength } \\
\mathrm{V}=\text { volume of the extract }(\mathrm{mL}) \\
\mathrm{W}=\text { weight of the fresh leaf tissue }(\mathrm{g})
\end{gathered}
$$

\subsection{Leaf Relative Water Content (LRWC)}

For the estimation of LRWC, the second one from top was used. In first step, after excising the leaf, the fresh weight was measured and tagged with a specific mark. Then, the leaf was soaked in $\mathrm{dH}_{2} \mathrm{O}$ for $4 \mathrm{~h}$. Then, the leaf was taken out of the water, it absorbed the extra surface water, and we measured its weight again and termed the result the turgid weight. The same leaf was then oven-dried at $75{ }^{\circ} \mathrm{C}$ for $48 \mathrm{~h}$ and again weighed and termed this the dry weight of leaf. Then LRWC was estimated using the formula from the obtained data

$$
\text { LRWC }(\%)=\frac{\text { Fresh weight of leaf }- \text { dry weight of leaf }}{\text { Turgid weight of leaf }- \text { dry weight of leaf }} \times 100
$$

\subsection{Leaf Relative Membrane Permeability}

We followed the method described by Yang et al. [51] to find out the leaf relative membrane permeability (LRMP). The known amount $(0.5 \mathrm{~g}$ ) of excised leaf was cut into small pieces (approximately $1 \mathrm{~cm}$ ) and put in test tubes having $20 \mathrm{~mL}$ of deionized $\mathrm{dH}_{2} \mathrm{O}$. After vortexing well for $5 \mathrm{~s}$, the EC of the assayed material was measured and termed as EC0. The test tubes containing leaf were then kept at $4^{\circ} \mathrm{C}$ for $24 \mathrm{~h}$, and the EC1 was measured. These test tubes containing leaf material were then autoclaved for $30 \mathrm{~min}$ at $120^{\circ} \mathrm{C}$ and assayed the EC2. The LRMP was measured using the following equation:

$$
\operatorname{RMP}(\%)=\frac{\mathrm{EC} 1-\mathrm{EC} 0}{\mathrm{EC} 2-\mathrm{EC} 0} \times 100
$$

\subsection{Estimation of Leaf Malondialdehyde Content}

Content of malondialdehyde (MDA) was measured using the method given by Cakmak and Horst [52] as the measure of lipid peroxidation. The trichloroacetic acid (TCA) method was used for the estimation of MDA content. One gram of freshly taken leaf material was ground in TCA ( $10 \%$ solution). The supernatant $(0.5 \mathrm{~mL})$ was obtained from the homogenized material and mixed with $3 \mathrm{~mL}$ of thiobarbituric acid (TBA), prepared in 20\% TCA. Test tubes having the triturate were kept at $95^{\circ} \mathrm{C}$ for $50 \mathrm{~min}$ and then cooled immediately in chilled water. After centrifugation $(10,000 \times \mathrm{g})$ of mixture for $10 \mathrm{~min}$, the absorbance of colored part was read at $600 \mathrm{~nm}$ and $532 \mathrm{~nm}$. The content of MDA was calculated using the following formula:

$$
\operatorname{MDA}(\mathrm{nmol})=\Delta(\mathrm{A} 532 \mathrm{~nm}-\mathrm{A} 600 \mathrm{~nm}) / 1.56 \times 105
$$

Absorption coefficient for the calculation of MDA is $156 \mathrm{mmol}^{-1} \mathrm{~cm}^{-1}$.

\subsection{Extraction of Antioxidant Enzymes and Total Soluble Proteins from Different Plant Parts}

For the extraction of antioxidant enzymes and total soluble proteins (TSP) from each plant part (root, stem, leaf), fresh material was ground $(0.5 \mathrm{~g})$ in chilled $(10 \mathrm{~mL}) 50-\mathrm{mM}$ phosphate buffer $(\mathrm{pH} 7.8)$. The mixture was then centrifuged at $10,000 \times g$ for $20 \mathrm{~min}$ at $4{ }^{\circ} \mathrm{C}$. The supernatant so obtained was then used for the estimation of total soluble proteins (TSP) and estimation of antioxidative enzymes activities. 


\subsubsection{Estimation of Total Soluble Proteins in Different Plant Parts}

TSP in the buffer extracts was estimated following the method of Bradford [53]. The absorbance of the triturate was measured at $595 \mathrm{~nm}$, and the quantities of the TSP in samples were computed using a series of protein standards $(200-1400 \mathrm{mg} / \mathrm{kg}$ ) prepared from analytical-grade bovine serum albumin (BSA).

2.7.2. Estimation of the Activities of Superoxide Dismutase, Peroxidase, and Catalase in Different Plant Parts

Activity of superoxide dismutase (SOD) was estimated using the method of Giannopolitis and Ries [54]. The method works based on the principle of photochemical reduction inhibition of nitroblue tetrazolium (NBT), which was used, and absorbance was read at $560 \mathrm{~nm}$ using an UV-visible spectrophotometer. However, the method of Chance and Maehly [55] was followed to measure the peroxidase (POD) and catalase (CAT) activities.

\subsection{Determination of Non-Enzymatic Antioxidants in Different Plant Parts}

Ascorbic acid (ASA) content in different plant parts was determined following Mukherjee and Choudhuri [56] after extraction in TCA. The flavonoid contents in different plant parts were determined following the methods ascribed by Karadeniz et al. [57]. However, the total tocopherol content in different plant parts was assayed following the method of Backer et al. [58]. The contents of ASA, flavonoids, and tocopherol were measured quantitively using the standard curves prepared with known concentration of analytical grade ASA, rutin, and $\alpha$-toc, respectively, obtained from Sigma-Aldrich Chemie GmbH - Schnelldorf, Germany.

\subsection{Determination Mineral Nutrients}

\subsubsection{Estimation of $\mathrm{K}^{+}, \mathrm{Ca}^{2+}$, and $\mathrm{Mg}^{2+}$ in Different Plan Parts}

For the estimation of mineral elements in different plant parts, $0.1 \mathrm{~g}$ dry material was digested using a $2 \mathrm{~mL}$ digestion mixture (prepared from $\mathrm{H}_{2} \mathrm{O}_{2}, \mathrm{H}_{2} \mathrm{SO}_{4}, \mathrm{LiSO}_{4}$, and Se metal). The final volume was maintained $50 \mathrm{~mL}$ using a volumetric flask. Flame photometer was used for determination of the contents of $\mathrm{K}^{+}$and $\mathrm{Ca}^{2+}$, while of $\mathrm{Mg}^{2+}$, contents were estimated using an Atomic Absorption Spectrophotometer (Hitachi, Model 7JO-8024, Tokyo, Japan).

\subsubsection{Determination of $\mathrm{N}$ and $\mathrm{P}$}

The nitrogen $(\mathrm{N})$ content from the digested material was determined following the method described by Bremner and Keeney [59]. The phosphorus (P) content from the digested material was estimated using Barton's reagent by spectrophotometrically, and quantity was estimated spectroscopically.

\subsection{Statistical Analysis}

Microsoft Excel software 2010, US was used for the estimation of means and standard errors from the collected. To find the significant differences among treatments, analysis of variance (ANOVA) was performed using Co-Stat window version 6.3, Cohorts, Berkeley, California, USA. To compare means for significant differences among treatments at $5 \%$ levels, Tukey's test (HSD-test) was performed. Correlations and PCA analysis were performed of the studied parameters using the XLSTAT software, version 2014.5, New York, USA and the significance among the generated values of each attribute was found using the Spearman's correlation table. 


\section{Results}

3.1. Different Growth Attributes and Content of Leaf Photosynthetic Pigments of Water-Stressed Maize Plants Foliar-Applied Alpha Tocopherol

Data for different morphological and growth attributes as presented in Table 1, which shows that water shortage imposed significant adverse impacts on the lengths of shoots and roots, the number of leaves, and the total leaf area of both maize cultivars (Table 2). Foliar application of $\alpha$-Toc significantly reduced the adverse impacts of water shortage on these growth attributes for both cultivars, and both wheat genotypes showed similar increasing response in this regard. However, root length and root fresh weights remained unaffected due to foliar spray of alpha tocopherols.

Reduced water supply significantly decreased the roots and shoots fresh and dry masses of both maize genotypes (Tables 1 and 2). Foliar spray of $\alpha$-Toc significantly reduced the adverse effects of water stress on these growth attributes. A similar increase in the root and shoot fresh and dry biomasses was found in both genotypes due to foliary-supplied $\alpha$-Toc, both under stressed and non-stressed conditions.

Leaf Chl. $a$, Chl. $b$, and total Chl. contents decreased significantly of both maize cultivars when grown under limited water supply. Both maize genotypes showed similar decreasing trend in leaf Chl. $a$, Chl. $b$, and total Chl. contents under drought stress. Significant increasing the effect of foliary-supplied $\alpha$-Toc was recorded on the contents of leaf Chl. $a, \mathrm{Chl} . b$, and total Chl. of both maize cultivars both under non-stressed and stressed conditions (Tables 2 and 3 ).

Chl. $a / b$ ratio was also significantly affected due to drought stress in both maize genotypes. An improvement in Chl. $a / b$ was recorded in cv. EV-1098, but the opposite was true for cv. Agaiti-2002. $\alpha$-Toc foliar spray significantly improved the leaf Chl. $a / b$ only in cv. Agaiti-2002 under conditions of limited water supply. However, the carotenoids content in different plant parts increased significantly due to water shortage in both maize genotypes (Tables 2 and 3), but this increase was cultivar and plant-part-specific. A significantly higher increase in carotenoids was found in leaf and root of cv. Agaiti-2002 in comparison to cv. EV-1098, but in relation with stem carotenoids content, this cultivar-specific difference was not found under drought stress. Foliar spray of $\alpha$-Toc further enhanced the content of carotenoids in all studied plant parts. Significantly more increase was recorded in the leaf and root of cv. Agaiti-2002 in comparison to cv. EV-1098. However, this improvement in stem carotenoids due to $\alpha$-Toc foliar application was same in both genotypes. Similar increasing trend in carotenoids under normal irrigation in all studied plant parts was also found in both genotypes due to $\alpha$-Toc foliar application (Tables 2 and 3). 
Table 1. Influence of foliar-applied alpha tocopherols on different growth and morphological attributes of maize cultivars grown under different water regimes (mean \pm SE; $n=3$ ). SL = shoot length; RL = root length; NOL = number of leaves; PLA = plant leaf area; SFW = shoot fresh weight; SDW = shoot dry weight; RFW = root fresh weight; RDW = root dry weight; $\alpha$-toc $=$ alpha tocopherol.

\begin{tabular}{|c|c|c|c|c|c|c|c|c|c|c|}
\hline & $\alpha$-Toc & Cultivars & SL (cm) & RL (cm) & NOL/plant & PLA $\left(\mathrm{cm}^{2}\right)$ & SFW (g) & SDW (g) & RFW (g) & RDW (g) \\
\hline \multirow{4}{*}{ Control } & \multirow{2}{*}{$0 \mathrm{mmol}$} & Agaiti-2002 & $12.23 \pm 1.10^{a}$ & $7.77 \pm 0.48^{a}$ & $3.75 \pm 0.20^{b}$ & $69.10 \pm 5.73^{b c}$ & $2.92 \pm 0.215^{b}$ & $0.37 \pm 0.04^{\mathrm{a}}$ & $1.18 \pm 0.15^{a}$ & $0.13 \pm 0.019^{b}$ \\
\hline & & EV-1098 & $12.03 \pm 0.67 \mathrm{a}$ & $6.70 \pm 0.50 \mathrm{ab}$ & $3.85 \pm 0.20^{b}$ & $62.40 \pm 4.04^{\mathrm{cd}}$ & $2.76 \pm 0.224 \mathrm{bc}$ & $0.32 \pm 0.03 \mathrm{abc}$ & $1.18 \pm 0.11^{\mathrm{a}}$ & $0.13 \pm 0.022^{b}$ \\
\hline & \multirow{2}{*}{$50 \mathrm{mmol}$} & Agaiti-2002 & $13.33 \pm 0.82^{a}$ & $6.20 \pm 0.35 \mathrm{bc}$ & $4.25 \pm 0.30^{\mathrm{a}}$ & $79.98 \pm 5.08^{\mathrm{a}}$ & $3.47 \pm 0.402^{\mathrm{a}}$ & $0.40 \pm 0.03 \mathrm{a}$ & $1.39 \pm 0.14^{a}$ & $0.16 \pm 0.014^{a}$ \\
\hline & & EV-1098 & $12.85 \pm 1.11^{a}$ & $7.50 \pm 0.79^{a}$ & $4.50 \pm 0.35^{a}$ & $73.60 \pm 0.63^{\mathrm{ab}}$ & $3.74 \pm 0.140^{\mathrm{a}}$ & $0.38 \pm 0.03^{\mathrm{a}}$ & $1.35 \pm 0.15^{a}$ & $0.17 \pm 0.024^{a}$ \\
\hline \multirow{5}{*}{ Drought } & \multirow[b]{2}{*}{$0 \mathrm{mmol}$} & Agaiti-2002 & $8.27 \pm 1.11^{\mathrm{d}}$ & $4.67 \pm 0.49^{c d}$ & $3.25 \pm 0.13^{c}$ & $45.50 \pm 1.06^{\mathrm{f}}$ & $2.28 \pm 0.145^{c}$ & $0.23 \pm 0.03^{b d}$ & $0.74 \pm 0.13^{c}$ & $0.09 \pm 0.021 \mathrm{~d}$ \\
\hline & & EV-1098 & $8.45 \pm 0.66^{\mathrm{d}}$ & $4.00 \pm 0.39 \mathrm{~d}$ & $3.00 \pm 0.35^{c}$ & $47.50 \pm 1.13^{\text {ef }}$ & $2.37 \pm 0.135^{c}$ & $0.24 \pm 0.01^{\mathrm{cd}}$ & $0.78 \pm 0.12^{c}$ & $0.10 \pm 0.011^{\mathrm{cd}}$ \\
\hline & \multirow{2}{*}{$50 \mathrm{mmol}$} & Agaiti-2002 & $10.30 \pm 1.51^{b}$ & $5.15 \pm 0.59 \mathrm{e}$ & $3.67 \pm 0.20^{b}$ & $55.00 \pm 1.74 \mathrm{de}$ & $2.56 \pm 0.136^{b}$ & $0.27 \pm 0.01^{\mathrm{cd}}$ & $0.93 \pm 0.16^{b}$ & $0.11 \pm 0.007 \mathrm{bcc}$ \\
\hline & & EV-1098 & $9.77 \pm 1.16^{c}$ & $4.73 \pm 0.37^{\mathrm{cd}}$ & $3.75 \pm 0.25^{b}$ & $54.50 \pm 1.07 \mathrm{def}$ & $2.72 \pm 0.139^{b}$ & $0.28 \pm 0.03 \mathrm{bcd}$ & $0.99 \pm 0.12^{b}$ & $0.12 \pm 0.018 \mathrm{bc}$ \\
\hline & LSD 5\% & & 1.311 & 1.11 & 0.414 & 4.26 & 0.50 & 0.081 & 0.15 & 0.025 \\
\hline
\end{tabular}

Values in column with same alphabets in superscript do not differ significantly.

Table 2. Mean squares from analysis of variance of the data for the studied attributes of water stressed maize plants foliar-applied with $\alpha$-Toc at seedling stage.

\begin{tabular}{|c|c|c|c|c|c|c|c|c|c|}
\hline SOV & d.f & SL & RL & NOL & LA & SFW & SDW & RFW & RDW \\
\hline WS & 1 & $66.7^{* * *}$ & $34.08^{* *}$ & $2.37^{* *}$ & $2440^{* * *}$ & $2.85^{* * *}$ & $0.045^{* *}$ & $1.06^{* * *}$ & $0.011^{* * *}$ \\
\hline Toc & 1 & $8.13^{* *}$ & $0.02 \mathrm{~ns}$ & $2.01 * *$ & $41.5^{* * *}$ & $1.67^{* *}$ & $0.003^{*}$ & $0.23 \mathrm{~ns}$ & $0.004^{* * *}$ \\
\hline $\mathrm{CV}$ & 1 & $0.53 \mathrm{~ns}$ & $0.42 \mathrm{~ns}$ & $0.01 \mathrm{~ns}$ & $37.5 *$ & $0.04 \mathrm{~ns}$ & $0.007 \mathrm{~ns}$ & $0.002 \mathrm{~ns}$ & $5.04 \times 10^{-4} \mathrm{~ns}$ \\
\hline WS * Toc & 1 & $0.54 \mathrm{~ns}$ & $0.88 \mathrm{~ns}$ & $0.02 \mathrm{~ns}$ & $8.16 \mathrm{~ns}$ & $0.34 \mathrm{~ns}$ & $0.004 \mathrm{~ns}$ & $6 \times 10^{-4} \mathrm{~ns}$ & $3.37 \times 10^{-4} \mathrm{~ns}$ \\
\hline $\mathrm{WS} * \mathrm{CV}$ & 1 & $0.16 \mathrm{~ns}$ & $0.66 \mathrm{~ns}$ & $0.17 \mathrm{~ns}$ & $121^{* * *}$ & $0.001 \mathrm{~ns}$ & $0.014 \mathrm{~ns}$ & $0.010 \mathrm{~ns}$ & $1.04 \times 10^{-4} \mathrm{~ns}$ \\
\hline $\mathrm{Toc}^{*} \mathrm{CV}$ & 1 & $0.67 \mathrm{~ns}$ & $1.92 \mathrm{~ns}$ & $0.09 \mathrm{~ns}$ & $0.17 \mathrm{~ns}$ & $0.08 \mathrm{~ns}$ & $0.002 \mathrm{~ns}$ & $2.7 \times 10^{-4} \mathrm{~ns}$ & $1.04 \times 10^{-4} \mathrm{~ns}$ \\
\hline $\mathrm{WS} * \mathrm{Toc} * \mathrm{CV}$ & 1 & $0.10 \mathrm{~ns}$ & $2.66 \mathrm{~ns}$ & $0.04 \mathrm{~ns}$ & $0.17 \mathrm{~ns}$ & $0.06 \mathrm{~ns}$ & $0.002 \mathrm{~ns}$ & $0.002 \mathrm{~ns}$ & $1.04 \times 10^{-4} \mathrm{~ns}$ \\
\hline Error & 16 & 0.78 & 3.31 & 0.17 & 7.08 & 0.13 & 0.003 & 0.065 & $2.17 \times 10^{-4}$ \\
\hline SOV & d.f & Protein $\mathrm{L}$ & Protein $\mathbf{R}$ & Potein S & LRWC & LRMP & Chl. $a$ & Chl. $b$ & Chl $a / b$ \\
\hline WS & 1 & $46728^{* * *}$ & $6834^{* * *}$ & $2281^{* * *}$ & $3314^{* * *}$ & $517^{* * *}$ & $0.15^{* * *}$ & $0.043^{* * *}$ & $1.270^{* * *}$ \\
\hline Toc & 1 & $11051^{* * *}$ & $3978^{* * *}$ & $253^{* *}$ & $172 * *$ & $50.0^{*}$ & $0.10^{* * *}$ & $0.015^{* * *}$ & $0.130 \mathrm{~ns}$ \\
\hline $\mathrm{CV}$ & 1 & $651.04^{* *}$ & $1395^{* *}$ & $13.5 \mathrm{~ns}$ & $118^{*}$ & $0.18 \mathrm{~ns}$ & $0.01 \mathrm{~ns}$ & $2.8 \times 10^{-4} \mathrm{~ns}$ & $0.004 \mathrm{~ns}$ \\
\hline WS * Toc & 1 & $35.04 \mathrm{~ns}$ & $234^{* * *}$ & $181.5^{* *}$ & $157^{* * *}$ & $76.5^{*}$ & $2.4 \times 10^{-6} \mathrm{~ns}$ & $0.004^{* *}$ & $0.290^{*}$ \\
\hline $\mathrm{WS} * \mathrm{CV}$ & 1 & $1717^{* * *}$ & $513^{* * *}$ & $37.5 \mathrm{~ns}$ & $22.6 \mathrm{~ns}$ & $0.80 \mathrm{~ns}$ & $4.8 \times 10^{-4} \mathrm{~ns}$ & $2.2 \times 10^{-5} \mathrm{~ns}$ & $0.003 \mathrm{~ns}$ \\
\hline $\mathrm{Toc}^{*} \mathrm{CV}$ & 1 & $1785^{* * *}$ & $18.4 \mathrm{~ns}$ & $37.5 \mathrm{~ns}$ & $1.25 \mathrm{~ns}$ & $16.1 \mathrm{~ns}$ & $0.002 \mathrm{~ns}$ & $5.8 \times 10^{-5} \mathrm{~ns}$ & $0.010 \mathrm{~ns}$ \\
\hline $\mathrm{WS} * \mathrm{Toc} * \mathrm{CV}$ & 1 & $3.37 \mathrm{~ns}$ & $408^{* * *}$ & $1.5 \mathrm{~ns}$ & $6.56 \mathrm{~ns}$ & $0.19 \mathrm{~ns}$ & $1.14 \times 10^{-54} \mathrm{~ns}$ & $0.002 *$ & $0.199 \mathrm{~ns}$ \\
\hline Error & 16 & 62.83 & 18 & 19.5 & 17.06 & 10.8 & 0.002 & $3.1 \times 10^{-4}$ & 0.051 \\
\hline
\end{tabular}


Table 2. Cont.

\begin{tabular}{|c|c|c|c|c|c|c|c|c|c|}
\hline SOV & d.f & Total Chl. & POD R & POD S & POD L & CAT R & CAT S & CAT L & SOD S \\
\hline WS & 1 & $0.351^{* * *}$ & $2223 * * *$ & $260 * * *$ & $1932 * * *$ & $1220^{* * *}$ & $329.8^{* * * *}$ & $2011^{* * *}$ & $270 * * *$ \\
\hline Toc & 1 & $0.190^{* * *}$ & $198^{* * *}$ & $37.6^{* * *}$ & $146^{* * *}$ & $359^{* * *}$ & $12.01 \mathrm{~ns}$ & $268^{* * *}$ & $4.74 \mathrm{~ns}$ \\
\hline $\mathrm{CV}$ & 1 & $0.008 \mathrm{~ns}$ & $135^{* *}$ & $149^{*}$ & $157^{* *}$ & $45.8^{* *}$ & $126.1^{* *}$ & $414^{* * *}$ & $71.4^{*}$ \\
\hline WS * Toc & 1 & $0.004 \mathrm{~ns}$ & $30.4 \mathrm{~ns}$ & $1.7 \times 10^{-4} \mathrm{~ns}$ & $0.89 \mathrm{~ns}$ & $18.6 \mathrm{~ns}$ & $1.054 \mathrm{~ns}$ & 0.219 * & $1.1 \mathrm{~ns}$ \\
\hline $\mathrm{WS} * \mathrm{CV}$ & 1 & $7 \times 10^{-4} \mathrm{~ns}$ & $9.4 \mathrm{~ns}$ & $1.50 \mathrm{~ns}$ & $1.21 \mathrm{~ns}$ & $3.2 \mathrm{~ns}$ & $12.07 \mathrm{~ns}$ & $19.65^{*}$ & $27.4 \mathrm{~ns}$ \\
\hline $\mathrm{Toc}^{*} \mathrm{CV}$ & 1 & $0.001 \mathrm{~ns}$ & $9.3 \mathrm{~ns}$ & $1.53 \mathrm{~ns}$ & $1.21 \mathrm{~ns}$ & $3.2 \mathrm{~ns}$ & $1.054 \mathrm{~ns}$ & $0.22 \mathrm{~ns}$ & $0.39 \mathrm{~ns}$ \\
\hline $\mathrm{WS} * \mathrm{Toc} * \mathrm{CV}$ & 1 & $0.003 \mathrm{~ns}$ & $3.4 \mathrm{~ns}$ & $5.93 \mathrm{~ns}$ & $0.08 \mathrm{~ns}$ & $3.5 \mathrm{~ns}$ & $1.032 \mathrm{~ns}$ & $2.85 \mathrm{~ns}$ & $0.018 \mathrm{~ns}$ \\
\hline Error & 16 & 0.002 & 9.5 & 7.10 & 12.1 & 6.9 & 8.286 & 8.91 & 8.485 \\
\hline SOV & d.f & SOD L & SOD R & MDA S & MDA L & MDA R & TOC S & TOC L & TOC R \\
\hline WS & 1 & $1229^{* * *}$ & $7222 * * *$ & $3174^{* * *}$ & $1890^{* * *}$ & $3313^{* * *}$ & $1493^{* * *}$ & $1666^{* * *}$ & $1741^{* * * *}$ \\
\hline Toc & 1 & $86.98^{*}$ & $176^{* *}$ & $73.5^{* *}$ & $828^{* * *}$ & $793 * * *$ & $83.7^{* *}$ & $1148^{* * *}$ & $489^{* * * *}$ \\
\hline $\mathrm{CV}$ & 1 & $81.40^{*}$ & $218^{* *}$ & $4.0^{*}$ & $84.3^{* * *}$ & $384^{* * *}$ & $9.0 \mathrm{~ns}$ & $60.11 *$ & $55.2^{*}$ \\
\hline WS * Toc & 1 & $107^{* *}$ & $24.7 \mathrm{~ns}$ & $1.5 \mathrm{~ns}$ & $108^{* * *}$ & $726^{* * *}$ & $3.24 \mathrm{~ns}$ & $170 * *$ & $1.30 \mathrm{~ns}$ \\
\hline $\mathrm{WS} * \mathrm{CV}$ & 1 & $0.06 \mathrm{~ns}$ & $16.1 \mathrm{~ns}$ & $6.0 \mathrm{~ns}$ & $18.3 \mathrm{~ns}$ & $337^{* * *}$ & $0.30 \mathrm{~ns}$ & $2.66 \mathrm{~ns}$ & $1.71 \mathrm{~ns}$ \\
\hline $\mathrm{Toc}^{*} \mathrm{CV}$ & 1 & $62.3 *$ & $0.05 \mathrm{~ns}$ & $1.5 \mathrm{~ns}$ & $30.3^{*}$ & $253^{* * *}$ & $0.42 \mathrm{~ns}$ & $0.16 \mathrm{~ns}$ & $1.71 \mathrm{~ns}$ \\
\hline $\mathrm{WS} * \mathrm{Toc}^{*} \mathrm{CV}$ & 1 & $28.8 \mathrm{~ns}$ & $0.80 \mathrm{~ns}$ & $1.5 \mathrm{~ns}$ & 18.3ns & $216^{* * *}$ & $3.24 \mathrm{~ns}$ & $0.66 \mathrm{~ns}$ & $0.01 \mathrm{~ns}$ \\
\hline Error & 16 & 10.9 & 15.65 & 4.9 & 5.12 & 8.62 & 5.52 & 12.0 & 11.3 \\
\hline SOV & d.f & AsA S & AsA L & AsA R & Car S & Car L & Car $\mathbf{R}$ & Flav S & Flav L \\
\hline WS & 1 & $14259^{* * * *}$ & $23814^{* * * *}$ & $26498^{* * *}$ & $5953^{* * *}$ & $10688^{* * * *}$ & $5730^{* * *}$ & 45.37 ** & $84.38^{* * *}$ \\
\hline Toc & 1 & $10533^{* * *}$ & $14113^{* * *}$ & $4858^{* * *}$ & $1722^{* * *}$ & $4776^{* * *}$ & $273 \mathrm{~ns}$ & $0.37 \mathrm{~ns}$ & $18.38 *$ \\
\hline $\mathrm{CV}$ & 1 & $630^{* * *}$ & $4056^{* * *}$ & $8720^{* * *}$ & $67.3 \mathrm{~ns}$ & $412.7^{*}$ & 894 * & $9.37 \mathrm{~ns}$ & $9.375 \mathrm{~ns}$ \\
\hline WS * Toc & 1 & $108^{*}$ & $937^{* * *}$ & $881^{* * *}$ & $146 \mathrm{~ns}$ & 265 & $8.77 \mathrm{~ns}$ & $0.38 \mathrm{~ns}$ & $3.35 \mathrm{~ns}$ \\
\hline $\mathrm{WS} * \mathrm{CV}$ & 1 & $63.3 \mathrm{~ns}$ & $0.01 \mathrm{~ns}$ & $930 * * *$ & $2.53 \mathrm{~ns}$ & 39.9 & $6.20 \mathrm{~ns}$ & $0.37 \mathrm{~ns}$ & $0.37 \mathrm{~ns}$ \\
\hline $\mathrm{Toc}^{*} \mathrm{CV}$ & 1 & $30.4 \mathrm{~ns}$ & $1.5 \mathrm{~ns}$ & $12.7 \mathrm{~ns}$ & $0.26 \mathrm{~ns}$ & 27.9 & $8.19 \mathrm{~ns}$ & $0.38 \mathrm{~ns}$ & $0.38 \mathrm{~ns}$ \\
\hline WS * $\mathrm{Toc}^{*} \mathrm{CV}$ & 1 & $30.3 \mathrm{~ns}$ & $253 *$ & $304^{* *}$ & $0.25 \mathrm{~ns}$ & 9.9 & $3.04 \mathrm{~ns}$ & $0.37 \mathrm{~ns}$ & $0.37 \mathrm{~ns}$ \\
\hline Error & 16 & 8.62 & 31.75 & 29.7 & 59.4 & 64.9 & 160.6 & 4.5 & 3.5 \\
\hline SOV & d.f & Flav R & $\mathrm{KS}$ & K L & K R & Ca S & $\mathrm{Ca} \mathrm{L}$ & Ca R & $\operatorname{Mg} \mathrm{S}$ \\
\hline WS & 1 & $135.4^{* * *}$ & $261^{* *}$ & $403^{* * *}$ & $396^{* * *}$ & $56.8^{* * *}$ & $172^{* * *}$ & $32.08^{* * *}$ & $0.014 \mathrm{~ns}$ \\
\hline Toc & 1 & $30.3^{*}$ & $53.2 \mathrm{~ns}$ & $68.2 *$ & $45.7 \mathrm{~ns}$ & $1.67 \mathrm{~ns}$ & $15.6^{* * *}$ & $14.7^{* *}$ & $0.144 \mathrm{~ns}$ \\
\hline $\mathrm{CV}$ & 1 & $9.37 \mathrm{~ns}$ & $2.83 \mathrm{~ns}$ & $1.82 \mathrm{~ns}$ & $0.55 \mathrm{~ns}$ & $2.66^{*}$ & $4.61^{*}$ & $0.26 \mathrm{~ns}$ & $0.768 \mathrm{~ns}$ \\
\hline
\end{tabular}


Table 2. Cont.

\begin{tabular}{|c|c|c|c|c|c|c|c|c|c|}
\hline WS * Toc & 1 & $3.375 \mathrm{~ns}$ & $0.15 \mathrm{~ns}$ & $2.95 \mathrm{~ns}$ & $0.40 \mathrm{~ns}$ & $6.36^{* *}$ & $0.90 \mathrm{~ns}$ & $0.011 \mathrm{~ns}$ & $1.264^{*}$ \\
\hline $\mathrm{WS}{ }^{*} \mathrm{CV}$ & 1 & $3.375 \mathrm{~ns}$ & $7.67 \mathrm{~ns}$ & $0.12 \mathrm{~ns}$ & $0.22 \mathrm{~ns}$ & $2.11 \mathrm{~ns}$ & $1.14 \mathrm{~ns}$ & $0.206 \mathrm{~ns}$ & $9 \times 10^{-4} \mathrm{~ns}$ \\
\hline $\mathrm{Toc} * \mathrm{CV}$ & 1 & $0.375 \mathrm{~ns}$ & $8.89 \mathrm{~ns}$ & $11.5 \mathrm{~ns}$ & $2.01 \mathrm{~ns}$ & $0.77 \mathrm{~ns}$ & $0.98 \mathrm{~ns}$ & $0.061 \mathrm{~ns}$ & $1.92 * *$ \\
\hline WS * $\mathrm{Toc}^{*} \mathrm{CV}$ & 1 & $0.375 \mathrm{~ns}$ & $0.81 \mathrm{~ns}$ & $0.26 \mathrm{~ns}$ & $1.02 \mathrm{~ns}$ & $0.85 \mathrm{~ns}$ & $0.14 \mathrm{~ns}$ & $0.160 \mathrm{~ns}$ & $0.069 \mathrm{~ns}$ \\
\hline Error & 16 & 4.125 & $20.1 \mathrm{~ns}$ & 11.9 & 14.2 & 0.57 & 0.79 & 1.035 & 0.221 \\
\hline SOV & d.f & Mg L & Mg R & P S & P L & P R & N S & N L & N R \\
\hline WS & 1 & $0.29 \mathrm{~ns}$ & $3.93^{* * *}$ & $10.5^{* * *}$ & $11.7^{* * *}$ & $5.15^{*}$ & $260^{* * *}$ & $321.84^{* * *}$ & $368.8^{* * *}$ \\
\hline Toc & 1 & $0.28 \mathrm{~ns}$ & $0.02 \mathrm{~ns}$ & $2.45^{*}$ & $1.15^{*}$ & $1.56 \mathrm{~ns}$ & $52.3 \mathrm{~ns}$ & $45.49 \mathrm{~ns}$ & $109.4^{*}$ \\
\hline $\mathrm{CV}$ & 1 & $2.86^{* * *}$ & $0.01 \mathrm{~ns}$ & $0.40 \mathrm{~ns}$ & $0.02 \mathrm{~ns}$ & $0.36 \mathrm{~ns}$ & $1.53 \mathrm{~ns}$ & $26.44 \mathrm{~ns}$ & $36.03 \mathrm{~ns}$ \\
\hline WS * Toc & 1 & $0.056 \mathrm{~ns}$ & $0.20 \mathrm{~ns}$ & $0.34 \mathrm{~ns}$ & $0.295 \mathrm{~ns}$ & $0.43 \mathrm{~ns}$ & $0.58 \mathrm{~ns}$ & $3.37 \mathrm{~ns}$ & $1.89 \mathrm{~ns}$ \\
\hline WS ${ }^{*} \mathrm{CV}$ & 1 & $2.95^{* * *}$ & $0.16 \mathrm{~ns}$ & $0.32 \mathrm{~ns}$ & $0.039 \mathrm{~ns}$ & $0.001 \mathrm{~ns}$ & $1.89 \mathrm{~ns}$ & $3.26 \mathrm{~ns}$ & $3.07 \mathrm{~ns}$ \\
\hline $\mathrm{Toc}^{*} \mathrm{CV}$ & 1 & $0.43 \mathrm{~ns}$ & $0.04 \mathrm{~ns}$ & $0.30 \mathrm{~ns}$ & $0.005 \mathrm{~ns}$ & $0.36 \mathrm{~ns}$ & $1.73 \mathrm{~ns}$ & $2.72 \mathrm{~ns}$ & $3.50 \mathrm{~ns}$ \\
\hline $\mathrm{WS} * \mathrm{Toc} * \mathrm{CV}$ & 1 & $1.23^{* *}$ & $0.12 \mathrm{~ns}$ & $1.11 \mathrm{~ns}$ & $0.324 \mathrm{~ns}$ & $0.35 \mathrm{~ns}$ & $1.81 \mathrm{~ns}$ & $1.52 \mathrm{~ns}$ & $2.14 \mathrm{~ns}$ \\
\hline Error & 16 & 0.11 & 0.13 & 0.51 & 0.203 & 0.69 & $21.4 \mathrm{~ns}$ & 11.65 & 19.3 \\
\hline
\end{tabular}

Table 3. Influence of foliar-applied alpha tocopherol on photosynthetic pigments of maize cultivars grown under different water regimes (mean \pm SE; $n=3)$. Chl. $a=$ leaf chlorophyll $a$; Chl. $b=$ leaf chlorophyll $b$; Chl $a / b=$ chlorophyll $a / b$ ratio; Total Chl. = total chlorophyll; Leaf Car = leaf carotenoids; Root Car = root carotenoids; Stem Car = stem carotenoids.

\begin{tabular}{|c|c|c|c|c|c|c|c|c|c|}
\hline Stress & $\alpha$-Toc & Cultivars & $\begin{array}{c}\text { Chl. } a \\
\text { (mg/g FW) }\end{array}$ & $\begin{array}{c}\text { Chl. b } \\
\text { (mg/g FW) }\end{array}$ & Chl $a / b$ & $\begin{array}{r}\text { Total Chl. } \\
\text { (mg/g FW) }\end{array}$ & $\begin{array}{c}\text { Leaf Car } \\
(\mu \mathrm{g} / \mathrm{g} \text { FW) }\end{array}$ & $\begin{array}{c}\text { Root Car } \\
(\mu \mathrm{g} / \mathrm{g} \text { FW) }\end{array}$ & $\begin{array}{r}\text { Stem Car } \\
(\mu \mathrm{g} / \mathrm{g} \text { FW }) \\
\end{array}$ \\
\hline \multirow{4}{*}{ Control } & \multirow[b]{2}{*}{$0 \mathrm{mmol}$} & Agaiti-2002 & $1.52 \pm 0.11^{\mathrm{ab}}$ & $0.36 \pm 0.01 \mathrm{bc}$ & $4.53 \pm 0.16^{\mathrm{a}}$ & $1.88 \pm 0.12^{\mathrm{ab}}$ & $102.16 \pm 1.78^{e}$ & $99.26 \pm 9.60$ de & $82.70 \pm 5.97 \mathrm{e}$ \\
\hline & & EV-1098 & $1.47 \pm 0.18 \mathrm{bc}$ & $0.38 \pm 0.02 \mathrm{bc}$ & $3.87 \pm 0.25^{d}$ & $1.85 \pm 0.17^{\mathrm{b}}$ & $93.52 \pm 4.41^{\mathrm{f}}$ & $84.16 \pm 12.26^{f}$ & $80.41 \pm 6.24 \mathrm{e}^{\mathrm{e}}$ \\
\hline & \multirow[b]{2}{*}{$50 \mathrm{mmol}$} & Agaiti-2002 & $1.63 \pm 0.06^{\mathrm{a}}$ & $0.47 \pm 0.01^{\mathrm{a}}$ & $4.40 \pm 0.14^{\mathrm{ab}}$ & $2.00 \pm 0.16^{\mathrm{ab}}$ & $120.27 \pm 13.12^{d}$ & $105.34 \pm 10.99^{d}$ & $105.00 \pm 2.83^{\mathrm{d}}$ \\
\hline & & EV-1098 & $1.60 \pm 0.14 \mathrm{ab}$ & $0.44 \pm 0.01 \mathrm{ab}$ & $3.64 \pm 0.33$ ed & $2.04 \pm 0.18^{\mathrm{a}}$ & $118.23 \pm 4.89^{\mathrm{d}}$ & $94.79 \pm 6.55^{\mathrm{e}}$ & $101.89 \pm 4.40^{\mathrm{d}}$ \\
\hline \multirow{5}{*}{ Drought } & \multirow{2}{*}{$0 \mathrm{mmol}$} & Agaiti-2002 & $1.34 \pm 0.04^{\mathrm{cd}}$ & $0.33 \pm 0.02^{c}$ & $3.62 \pm 0.09^{\mathrm{e}}$ & $1.67 \pm 0.05^{b}$ & $139.98 \pm 10.40^{\mathrm{b}}$ & $129.65 \pm 9.31 \mathrm{ab}$ & $120.00 \pm 5.83^{b}$ \\
\hline & & EV-1098 & $1.31 \pm 0.12 \mathrm{bc}$ & $0.31 \pm 0.02^{c}$ & $4.28 \pm 0.05^{b c}$ & $1.62 \pm 0.10^{c}$ & $127.25 \pm 5.61^{c}$ & $118.75 \pm 2.98^{c}$ & $116.00 \pm 5.75^{c}$ \\
\hline & \multirow{3}{*}{$50 \mathrm{mmol}$} & Agaiti-2002 & $1.46 \pm 0.12 \mathrm{bcd}$ & $0.34 \pm 0.02^{c}$ & $4.29 \pm 0.16^{b c}$ & $1.80 \pm 0.10^{b}$ & $176.08 \pm 2.74 \mathrm{a}$ & $134.73 \pm 11.52^{\mathrm{a}}$ & $132.00 \pm 6.54 \mathrm{a}$ \\
\hline & & EV-1098 & $1.46 \pm 0.09 \mathrm{bcd}$ & $0.35 \pm 0.02^{c}$ & $4.17 \pm 0.16^{c}$ & $1.81 \pm 0.12^{b}$ & $163.33 \pm 5.61^{b}$ & $124.26 \pm 9.60 \mathrm{~b}^{\mathrm{c}}$ & $128.00 \pm 4.89 \mathrm{al}$ \\
\hline & & & 0.15 & 0.12 & 0.64 & 0.52 & 8.74 & 11.81 & 8.15 \\
\hline
\end{tabular}

Values in column with same alphabets in superscript do not differ significantly. 
3.2. Leaf Relative Water Content, Leaf Relative Membrane Permeability, Total Soluble Proteins, and $\mathrm{H}_{2} \mathrm{O}_{2}$ Contents of Leaf Photosynthetic Pigments of Maize Plants Foliar-Applied with Alpha Tocopherol

Data presented in Table 3 reveals that the imposition of water stress decreased the LRWC of both genotypes, and a slightly higher decrease in LRWC was found in cv. EV-1098 in comparison to cv. Agaiti-2002. The foliar application of $\alpha$-Toc significantly increased the LRWC of both genotypes, and this increase was found only under drought-stressed conditions; both cultivars showed a similar increasing trend in this regard (Tables 2 and 4 ).

Leaf relative membrane permeability (LRMP) increased significantly under water deficit conditions, and this increase was similar in both maize cultivars. Exogenous application of $\alpha$-Toc as foliar spray was found to be effective in decreasing the LRMP in both maize cultivars under water-stressed conditions, and both maize cultivars showed similar responses in this regard (Tables 2 and 4).

Drought stress exerted a tissue-specific increment in leaf, root, and stem TSP contents of both genotypes when grown without foliar application of $\alpha$-Toc. In leaf and root, this improvement in TSP was higher in cv. Agaiti-2002 in comparison to cv. EV-1098, but in relation to stem TSP, both cultivars showed the same increasing trend. Exogenous application of $\alpha$-Toc further improved TSP accumulation in all studied plant parts in both maize cultivars under stressed and non-stressed conditions. Alpha-toc-induced this improvement in TSP contents was significantly more prominent in leaves of cv. Agaiti-2002 in comparison to cv. EV-1098 under limited water supply, but a similar increasing trend was recorded in root and stem (Tables 2 and 4).

Under stressful conditions, the extent of oxidative damage is measured in terms of MDA contents. The data presented shows that MDA contents in all studied plant parts of both the cultivars increased significantly under limited water supply. $\alpha$-Toc foliar-application significantly reduced the MDA accumulation in all studied plant parts, and a more prominent reduction was found in leaves in comparison to other plant parts in both maize genotypes (Tables 2 and 4). 
Table 4. Influence of foliar application of $\alpha$-Toc on leaf relative water content, leaf relative membrane permeability, tissue specific total soluble proteins and malondialdehyde content of maize cultivars grown under different water regimes (mean $\pm \mathrm{SE} ; n=3$ ). LRWC = leaf relative water content; RMP $=$ leaf relative membrane permeability; Leaf TSP = leaf total soluble proteins; Root TSP = root total soluble proteins; Stem TSP = stem total soluble proteins; Leaf MDA = leaf malondialdehyde; Root MDA = root malondialdehyde; Stem MDA = stem malondialdehyde.

\begin{tabular}{|c|c|c|c|c|c|c|c|c|c|c|}
\hline Stress & $\alpha$-Toc & Cultivars & LRWC (\%) & RMP (\%) & $\begin{array}{c}\text { Leaf TSP } \\
\text { (mg/kg FW) }\end{array}$ & $\begin{array}{c}\text { Root TSP } \\
\text { (mg/kg FW) }\end{array}$ & $\begin{array}{c}\text { Stem TSP } \\
\text { (mg/kg FW) }\end{array}$ & $\begin{array}{c}\text { Leaf MDA } \\
\text { (nmol/g FW) }\end{array}$ & $\begin{array}{c}\text { Root MDA } \\
\text { (nmol/g FW) }\end{array}$ & $\begin{array}{c}\text { Stem MDA } \\
\text { (nmol/g FW) }\end{array}$ \\
\hline \multirow{4}{*}{ Control } & \multirow{2}{*}{$0 \mathrm{mmol}$} & Agaiti-2002 & $87.54 \pm 1.71^{\mathrm{a}}$ & $32.33 \pm 3.92 \mathrm{e}$ & $210.00 \pm 29.89^{f}$ & $144.33 \pm 7.50^{\mathrm{f}}$ & $73.00 \pm 15.15^{c}$ & $68.10 \pm 1.19 \mathrm{bc}$ & $66.18 \pm 6.40^{c}$ & $62.02 \pm 4.48^{\mathrm{cc}}$ \\
\hline & & EV-1098 & $81.73 \pm 5.00^{b}$ & $33.96 \pm 2.33$ de & $233.33 \pm 23.34^{\mathrm{e}}$ & $160.33 \pm 4.58^{\mathrm{e}}$ & $72.00 \pm 9.36^{c}$ & $62.35 \pm 2.94^{c}$ & $66.10 \pm 8.17^{c}$ & $60.31 \pm 5.18^{\mathrm{cc}}$ \\
\hline & \multirow{2}{*}{$50 \mathrm{mmol}$} & Agaiti-2002 & $88.36 \pm 1.62^{a}$ & $34.83 \pm 2.76^{\mathrm{d}}$ & $267.33 \pm 24.22 \mathrm{~d}$ & $186.67 \pm 2.57^{\mathrm{cd}}$ & $83.67 \pm 9.01^{b}$ & $60.18 \pm 8.75^{c}$ & $65.23 \pm 7.33^{c}$ & $60.35 \pm 2.12^{c}$ \\
\hline & & EV-1098 & $81.38 \pm 5.36^{b}$ & $33.55 \pm 3.19$ de & $257.00 \pm 18.26^{\mathrm{e}}$ & $182.00 \pm 3.45^{\mathrm{d}}$ & $86.67 \pm 14.81^{b}$ & $55.49 \pm 3.26$ bc & $66.53 \pm 4.37^{c}$ & $56.42 \pm 3.29^{c}$ \\
\hline \multirow{5}{*}{ Drought } & \multirow[b]{2}{*}{$0 \mathrm{mmol}$} & Agaiti-2002 & $58.23 \pm 3.52^{d}$ & $45.32 \pm 0.50^{\mathrm{b}}$ & $312.33 \pm 31.67^{c}$ & $183.67 \pm 10.05^{\mathrm{d}}$ & $101.67 \pm 16.96^{a}$ & $90.65 \pm 6.94^{\text {a }}$ & $99.83 \pm 6.21^{\mathrm{a}}$ & $85.59 \pm 4.37$ at \\
\hline & & EV-1098 & $51.34 \pm 3.09^{e}$ & $47.88 \pm 3.78^{a}$ & $304.67 \pm 25.01^{c}$ & $201.33 \pm 5.64^{b}$ & $94.33 \pm 13.58^{\mathrm{ab}}$ & $84.84 \pm 3.74^{a}$ & $102.84 \pm 1.98^{\mathrm{a}}$ & $84.04 \pm 4.32 \mathrm{~b}$ \\
\hline & \multirow[b]{2}{*}{$50 \mathrm{mmol}$} & Agaiti-2002 & $67.87 \pm 0.62^{c}$ & $40.29 \pm 2.08^{c}$ & $376.67 \pm 22.30^{\mathrm{a}}$ & $196.00 \pm 9.98 \mathrm{bc}$ & $99.00 \pm 9.93^{\mathrm{a}}$ & $70.72 \pm 1.83^{b}$ & $64.3 \pm 7.68^{\mathrm{b}}$ & $81.92 \pm 4.91$ at \\
\hline & & EV-1098 & $66.17 \pm 2.02^{c}$ & $39.08 \pm 2.44^{\mathrm{c}}$ & $330.67 \pm 33.06^{b}$ & $227.67 \pm 5.81^{a}$ & $98.00 \pm 10.98^{a}$ & $72.22 \pm 3.74^{b}$ & $92.50 \pm 6.40^{b}$ & $80.60 \pm 3.67^{a}$ \\
\hline & LSD 5\% & & 4.06 & 3.49 & 24.44 & 12.64 & 10.26 & 5.82 & 7.88 & 6.12 \\
\hline
\end{tabular}

Values in column with same alphabets in superscript do not differ significantly. 
3.3. Root, Stem, and Leaf Total Tocopherol (Figure 1A-C); Ascorbic Acid (Figure 1D-F); and Total Flavonoid Contents (Figure 1G-I) of Maize Plants Foliar-Applied with $\alpha$-Toc

Imposition of water stress significantly increased the accumulation of total Toc contents in the studied plant parts of both maize cultivars. This accumulation in total-Toc content in all studied plant parts was increased further due to the foliar application of $\alpha$-Toc. This increased accumulation in internal total-Toc in all studied plant parts due to its foliar application was more in root and leaf in comparison to stem in both genotypes under both non-stressed and stressed conditions. $\alpha$-Toc applied this increase in all studied plant parts and was similar in both maize cultivars (Figure 1A-C).
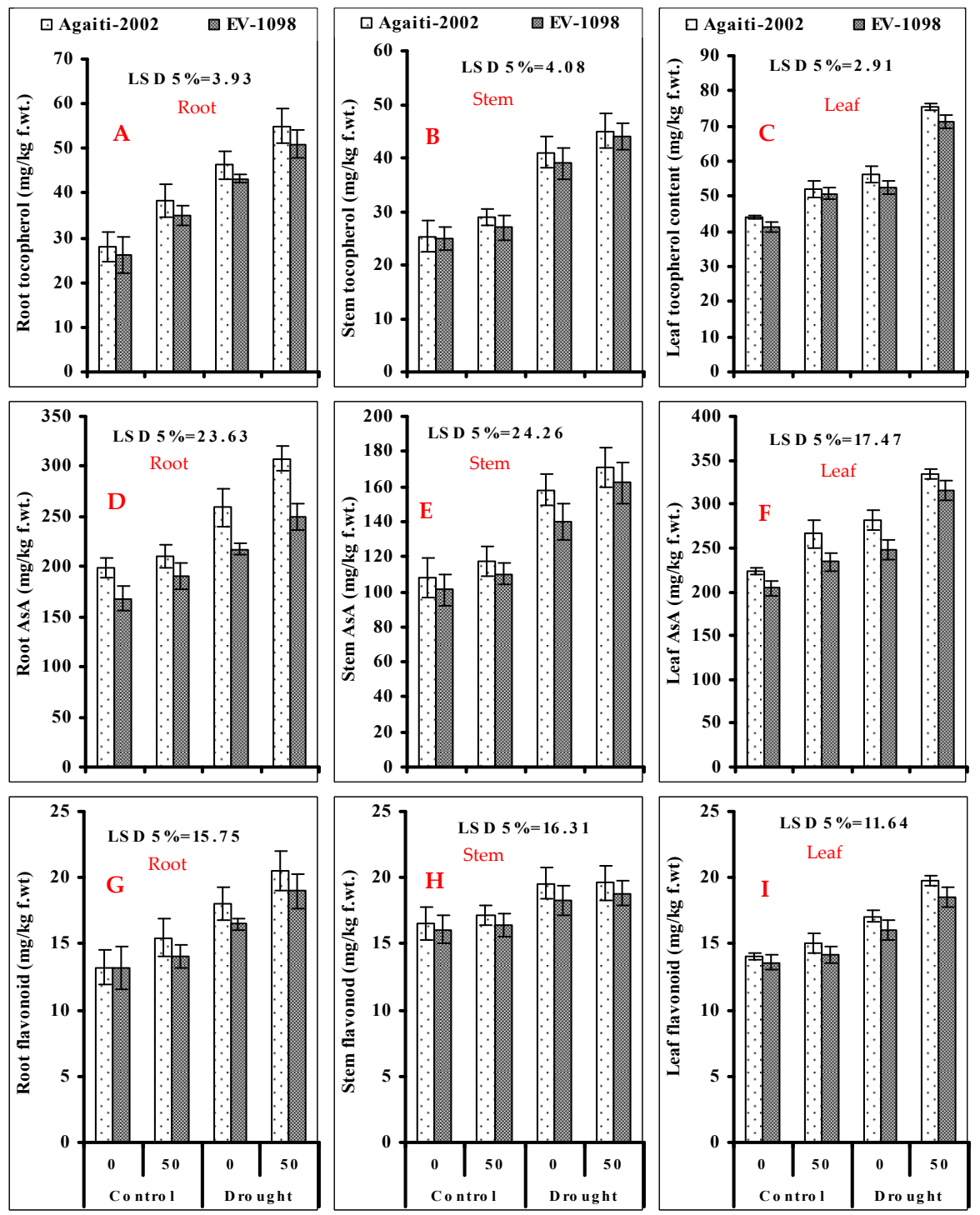

Figure 1. Root, stem, and leaf total-Toc (A-C), AsA (D-F), and total flavonoids (G-I) of maize plants foliar-applied with $\alpha$-Toc when grown under water deficit conditions (mean $\pm \mathrm{SE}$; $n=4$ ); AsA $=$ ascorbic acid; 0 and $50=$ mmol solution of $\alpha$-Tocopherol for foliar spray.

AsA and flavonoid contents in different studied plant parts also increased significantly in both genotypes under water deficit conditions, and this improvement in AsA and flavonoid accumulation was more in root and leaf in cv. Agaiti-2002 in comparison to cv. EV-1098 (Figure 1; Table 2). Exogenous application of $\alpha$-Toc as foliar spray further enhanced the AsA accumulation in all studied plant parts of both maize genotypes; accumulation was higher in cv. Agaiti-2002, both under stressed and non-stressed conditions. However, improvement in flavonoids was found only in the leaf and root 
of both maize genotypes when grown under water deficit conditions; this improved accumulation in flavonoids was not found in stem flavonoids (Figure 1D-I).

\subsection{Activities of CAT (Figure 2A-C), SOD (Figure 2D-F) and POD (2G-I) in Root, Stem, and Leaf of Maize Plants Foliar-Applied with $\alpha$-Toc}

Activities of CAT and SOD in all studied plant parts increased significantly in both genotypes when grown under limited water supply, and comparatively more improvement was found in root and leaf of cv. Agaiti-2002 in comparison with stem. Alpha-toc application further enhanced the CAT and SOD activities in root and leaf in both genotypes, but such improvement in CAT and SOD activities was not found in the stem of both genotypes. In leaf, significantly more improvement in CAT activity due to $\alpha$-Toc application was recorded in cv. Agaiti-2002 as compared with cv. EV-1098; however, in relation with SOD activity in root and leaf, cv. Agaiti-2002 was superior in comparison to cv. EV-1098 due to $\alpha$-Toc application (Figure 2A-F; Table 2).
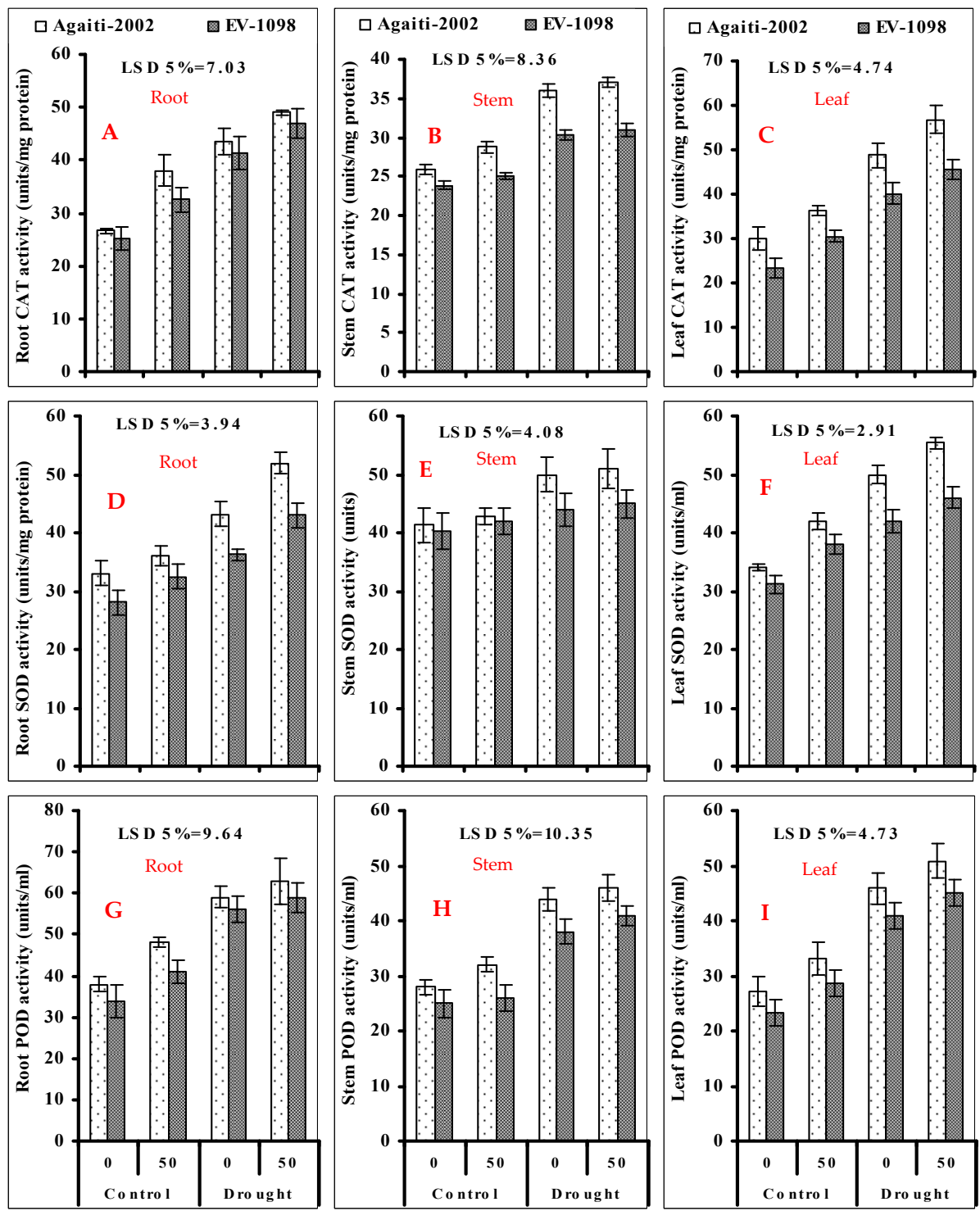

Figure 2. Activities of CAT (A-C), SOD (D-F), and POD (G-I) in root, stem and leaf, respectively, of drought-stressed maize plants applied with $\alpha$-Toc as foliar spray when grown under water deficit conditions (mean $\pm \mathrm{SE} ; n=4$ ). $\mathrm{CAT}=$ catalase; $\mathrm{SOD}=$ superoxide dismutase; $\mathrm{POD}=$ peroxidase 0 and $50=$ mmol solution of $\alpha$-Tocopherol for foliar spray. 
Like CAT and SOD activities, POD activity was also improved significantly under limited water supply in both genotypes in all studied plant parts. Foliar spray of $\alpha$-Toc further enhanced the POD activity in root and leaf of both genotypes under non-stressed and stressed conditions, but this improvement in POD activity was not found in stem of both genotypes. A non-significant difference between genotypes was found in this regard (Figure 2G-I; Table 2).

\subsection{Contents of $\mathrm{K}, \mathrm{Ca}, \mathrm{Mg}, \mathrm{N}$, and P in Different Parts of Maize Plants Foliary-Applied with $\alpha$-Toc When} Grown under Different Water Regimes

Drought stress significantly altered the tissue-specific acquisition patterns of macro-nutrients of both the studied cultivars when grown without $\alpha$-Toc application (Tables 2 and 5). Potassium contents of leaf, root, and stem were reduced significantly grown under water stress without foliar spray of $\alpha$-Toc. Foliar application of $\alpha$-Toc increased the potassium content in specific organs under non-stressed and stressed conditions, and the impact was significant for leaf and root K of cv. Agaiti-2002 under water stress. The leaf, root and stem $\mathrm{Ca}$ and $\mathrm{Mg}$ uptake was also significantly improved after foliar application of $\alpha$-Toc in both maize genotypes under non-stressed and stressed conditions, which was impaired due to limited water supply. This prominent difference in the uptake of $\mathrm{K}^{+}, \mathrm{Ca}^{2+}$, and $\mathrm{Mg}^{2+}$ due to $\alpha$-Toc foliar application was similar in both maize genotypes under non-stressed and stressed conditions.

Like other nutrients, drought stress also negatively affected the P and $\mathrm{N}$ uptake in leaf, root, and stem of both the cultivars and this impact was more prominent on leaf and stem N. Exogenous application of $\alpha$-Toc helped both the cultivars to maintain their $N$ and P nutrition of root, leaf and stem under non-stressed and stressed conditions. Regarding the N contents in studied plant parts, comparatively more improvement in $\mathrm{N}$ uptake due to $\alpha$-Toc foliar spray was found in leaf and root than stem (Tables 2 and 5). 
Table 5. Influence of foliar-applied $\alpha$-Toc on tissue specific organic and inorganic minerals of two maize (Zea mays L.) cultivars under non-stress and water-stressed conditions (mean $\pm \mathrm{SE} ; n=3$ ).

\begin{tabular}{|c|c|c|c|c|c|c|c|c|c|c|c|}
\hline Stress & $\alpha$-Toc & Cultivars & $\begin{array}{c}\mathrm{K}^{+} \text {Leaf } \\
\left(\mathrm{mg} \mathrm{g}^{-1} \mathrm{DW}\right)\end{array}$ & $\begin{array}{c}\mathrm{K}^{+} \text {Root } \\
\left(\mathrm{mg} \mathrm{g}^{-1} \mathrm{DW}\right)\end{array}$ & $\begin{array}{c}\mathrm{K}^{+} \text {stem } \\
\left(\mathrm{mg} \mathrm{g}^{-1} \mathrm{DW}\right)\end{array}$ & $\begin{array}{c}\mathrm{Ca}^{2+} \text { leaf } \\
\left(\mathrm{mg} \mathrm{g}^{-1} \mathrm{DW}\right)\end{array}$ & $\begin{array}{c}\mathrm{Ca}^{2+} \text { Root } \\
\left(\mathrm{mg} \mathrm{g}^{-1} \mathrm{DW}\right)\end{array}$ & $\begin{array}{c}\mathrm{Ca}^{2+} \text { stem } \\
\left(\mathrm{mg} \mathrm{g}^{-1} \mathrm{DW}\right)\end{array}$ & $\begin{array}{c}\mathrm{Mg}^{2+} \text { leaf } \\
\left(\mathrm{mg} \mathrm{g}^{-1} \mathrm{DW}\right)\end{array}$ & $\begin{array}{c}\mathrm{Mg}^{2+} \text { root } \\
\left(\mathrm{mg} \mathrm{g}^{-1} \mathrm{DW}\right)\end{array}$ & $\begin{array}{c}\mathrm{Mg}^{2+} \text { stem } \\
\left(\mathrm{mg} \mathrm{g}^{-1} \mathrm{DW}\right)\end{array}$ \\
\hline \multirow{4}{*}{ Control } & \multirow{2}{*}{$0 \mathrm{mmol}$} & Agaiti-2002 & $39.30 \pm 3.18^{a}$ & $41.35 \pm 2.99 \mathrm{ab}$ & $33.09 \pm 3.20 \mathrm{bc}$ & $11.35 \pm 0.20^{\mathrm{d}}$ & $10.34 \pm 0.75^{c}$ & $10.03 \pm 0.61^{b}$ & $3.41 \pm 0.06^{\mathrm{b}}$ & $4.13 \pm 0.30^{\mathrm{a}}$ & $3.31 \pm 0.32 \mathrm{ab}$ \\
\hline & & EV-1098 & $41.17 \pm 1.47 \mathrm{ab}$ & $40.21 \pm 2.12^{\mathrm{b}}$ & $31.99 \pm 2.38^{c}$ & $12.92 \pm 0.61^{\mathrm{c}}$ & $10.05 \pm 0.03^{c}$ & $9.02 \pm 0.43^{c}$ & $2.73 \pm 0.15^{d}$ & $4.02 \pm 0.12^{\mathrm{a}}$ & $3.14 \pm 0.31^{b}$ \\
\hline & \multirow[b]{2}{*}{$50 \mathrm{mmol}$} & Agaiti-2002 & $43.15 \pm 2.19^{a}$ & $42.90 \pm 1.41^{a}$ & $35.12 \pm 1.67 \mathrm{ab}$ & $14.36 \pm 1.46^{b}$ & $10.99 \pm 0.18^{b}$ & $11.71 \pm 1.22 \mathrm{a}$ & $4.01 \pm 0.44^{\mathrm{a}}$ & $4.29 \pm 0.14^{\mathrm{a}}$ & $3.51 \pm 0.33^{\mathrm{a}}$ \\
\hline & & EV-1098 & $43.67 \pm 2.45^{a}$ & $42.93 \pm 1.77^{a}$ & $36.00 \pm 1.21^{\mathrm{a}}$ & $15.33 \pm 0.40^{a}$ & $12.23 \pm 0.31^{\mathrm{a}}$ & $10.40 \pm 0.36^{b}$ & $3.27 \pm 0.16^{b c}$ & $4.09 \pm 0.22 \mathrm{a}$ & $3.45 \pm 0.07^{\mathrm{a}}$ \\
\hline \multirow{5}{*}{ Drought } & \multirow{2}{*}{$0 \mathrm{mmol}$} & Agaiti-2002 & $30.33 \pm 3.47^{\mathrm{e}}$ & $35.67 \pm 2.82 \mathrm{~cd}$ & $25.00 \pm 1.85$ ef & $6.48 \pm 0.59 \mathrm{~g}$ & $7.33 \pm 0.40^{\mathrm{e}}$ & $5.74 \pm 0.71^{\mathrm{g}}$ & $2.03 \pm 0.35^{\mathrm{e}}$ & $2.64 \pm 0.29 \mathrm{~b}$ & $2.38 \pm 0.22 \mathrm{~d}$ \\
\hline & & EV-1098 & $32.33 \pm 1.76$ de & $33.00 \pm 2.79 \mathrm{e}$ & $24.33 \pm 1.76^{\mathrm{f}}$ & $7.67 \pm 0.40^{f}$ & $7.67 \pm 0.81^{\mathrm{f}}$ & $6.87 \pm 0.72^{f}$ & $2.24 \pm 0.19^{\mathrm{e}}$ & $2.34 \pm 0.29 b$ & $2.82 \pm 0.26^{c}$ \\
\hline & & Agaiti-2002 & $36.00 \pm 1.85^{c}$ & $37.64 \pm 2.29^{c}$ & $27.33 \pm 1.76^{d}$ & $8.45 \pm 0.30^{\mathrm{e}}$ & $9.15 \pm 0.82^{\mathrm{d}}$ & $8.33 \pm 0.40^{\mathrm{e}}$ & $2.54 \pm 0.09^{d}$ & $2.66 \pm 0.33^{b}$ & $2.49 \pm 0.31 \mathrm{~d}$ \\
\hline & $50 \mathrm{mmol}$ & EV-1098 & $34.81 \pm 2.19^{\mathrm{cd}}$ & $34.67 \pm 2.45$ de & $27.00 \pm 3.89$ de & $8.33 \pm 0.40^{\mathrm{e}}$ & $9.96 \pm 0.66^{c}$ & $8.88 \pm 0.13^{\mathrm{d}}$ & $3.11 \pm 0.19^{c}$ & $2.71 \pm 0.25^{b}$ & $2.47 \pm 0.20^{d}$ \\
\hline & LSD $5 \%$ & & 2.99 & 2.20 & 2.26 & 0.50 & 0.47 & 0.40 & 0.29 & 0.41 & 0.22 \\
\hline Stress & $\alpha$-Toc & Cultivars & $\begin{array}{c}\mathrm{N} \text { Leaf } \\
\left(\mathrm{mg} \mathrm{g}^{-1} \mathrm{DW}\right)\end{array}$ & $\begin{array}{c}\text { N Root } \\
\left(\mathrm{mg} \mathrm{g}^{-1} \text { DW }\right)\end{array}$ & $\begin{array}{c}\mathrm{N} \text { Stem } \\
\left(\mathrm{mg} \mathrm{g}^{-1} \mathrm{DW}\right)\end{array}$ & $\begin{array}{c}\text { P Leaf } \\
\left(\mathrm{mg} \mathrm{g}^{-1} \mathrm{DW}\right)\end{array}$ & $\begin{array}{c}\text { P Root } \\
\left(\mathrm{mg} \mathrm{g}^{-1} \text { DW) }\right.\end{array}$ & $\begin{array}{c}\text { P Stem } \\
\left(\mathrm{mg} \mathrm{g}^{-1} \mathrm{DW}\right)\end{array}$ & & & \\
\hline \multirow{4}{*}{ Control } & \multirow[b]{2}{*}{$0 \mathrm{mmol}$} & Agaiti-2002 & $38.63 \pm 2.28^{b}$ & $41.35 \pm 2.99 \mathrm{ab}$ & $33.09 \pm 3.20^{b}$ & $5.20 \pm 0.33^{b}$ & $5.91 \pm 0.43^{b}$ & $4.50 \pm 0.46^{\mathrm{c}}$ & & & \\
\hline & & EV-1098 & $41.17 \pm 1.47^{\mathrm{b}}$ & $40.21 \pm 2.12 \mathrm{ab}$ & $32.72 \pm 2.93^{b}$ & $5.55 \pm 0.25^{b}$ & $5.74 \pm 0.27^{b}$ & $4.01 \pm 0.28 \mathrm{de}$ & & & \\
\hline & \multirow{3}{*}{$50 \mathrm{mmol}$} & Agaiti-2002 & $45.82 \pm 2.77^{a}$ & $42.90 \pm 1.41^{\mathrm{a}}$ & $38.16 \pm 1.90^{\mathrm{a}}$ & $6.06 \pm 0.34 \mathrm{a}$ & $6.13 \pm 0.20 \mathrm{ab}$ & $5.02 \pm 0.22^{b}$ & & & \\
\hline & & EV-1098 & $47.00 \pm 2.52^{\mathrm{a}}$ & $43.93 \pm 2.72^{\mathrm{a}}$ & $39.06 \pm 2.73^{a}$ & $6.01 \pm 0.23^{\mathrm{a}}$ & $6.28 \pm 0.31^{\mathrm{a}}$ & $5.28 \pm 0.49^{\mathrm{a}}$ & & & \\
\hline \multirow{5}{*}{ Drought } & & Agaiti-2002 & $25.33 \pm 3.46^{\mathrm{d}}$ & $33.33 \pm 2.45^{c}$ & $26.00 \pm 2.27^{c}$ & $4.33 \pm 0.50^{\mathrm{cd}}$ & $4.62 \pm 0.42^{\mathrm{d}}$ & $3.84 \pm 0.22 \mathrm{e}$ & & & \\
\hline & $0 \mathrm{mmol}$ & EV-1098 & $23.33 \pm 2.64^{d}$ & $34.41 \pm 3.00 \mathrm{e}$ & $23.00 \pm 3.20^{d}$ & $4.06 \pm 0.27^{d}$ & $4.86 \pm 0.50^{\mathrm{cd}}$ & $3.58 \pm 0.25^{\mathrm{f}}$ & & & \\
\hline & \multirow[b]{2}{*}{$50 \mathrm{mmol}$} & Agaiti-2002 & $30.00 \pm 2.10^{c}$ & $36.61 \pm 3.27^{b}$ & $31.00 \pm 1.85^{b}$ & $4.29 \pm 0.27^{\mathrm{cd}}$ & $5.02 \pm 0.47^{c}$ & $4.08 \pm 0.23^{\mathrm{d}}$ & & & \\
\hline & & EV-1098 & $32.67 \pm 0.81^{\mathrm{c}}$ & $37.67 \pm 1.45^{b}$ & $27.67 \pm 2.02^{\mathrm{c}}$ & $4.54 \pm 0.22^{\mathrm{c}}$ & $5.05 \pm 0.53^{c}$ & $3.82 \pm 0.11^{\mathrm{e}}$ & & & \\
\hline & LSD $5 \%$ & & 2.95 & 4.00 & 2.80 & 0.39 & 0.32 & 0.22 & & & \\
\hline
\end{tabular}

Values in column with same alphabets in superscript do not differ significantly. 
3.6. PCA Analysis and Spearman's Correlation Coefficient $\left(r^{2}\right)$ Values Extracted from XLSTAT Software of All the Studied Attributes of Maize Plants Foliar-Applied with $\alpha$-Toc

PCA and correlations coefficients among studied attributes revealed a significant positive correlation of total-Toc contents in leaf, root, and stem with morphological and growth attributes, levels of antioxidants, and uptake of mineral nutrients $(\mathrm{K}, \mathrm{Ca}, \mathrm{Mg}, \mathrm{N}$, and $\mathrm{P})$ in all studied tissues of maize. A positive correlation of leaf and stem Toc was found with leaf area $\left(0.768^{* * *}\right.$ and $\left.0.664^{* *}\right)$ and fresh weights $\left(0.921^{* * *}\right.$ and $\left.0.661^{* * *}\right)$, respectively, that depicts the role of Toc in the improved growth under drought stress. Positive correlation was also recorded of shoot dry weight with Toc levels in studied plant tissues such as in leaf $\left(0.578^{* *}\right)$ and root $\left(0.643^{* * *}\right)$, respectively. Significantly positive correlation was found of Toc levels in the root with LRWC $\left(0.721^{* * *}\right)$. CAT, POD, and SOD activities in different plant parts like leaf $\left(0.966^{* * *}, 0.961^{* * *}\right.$ and $\left.0.936^{* * *}\right)$ and stem $\left(0.863^{* * *}, 0.872^{* * *}\right.$ and $\left.0.859^{* * *}\right)$, respectively, were also positively correlated with plant Toc levels. Tocopherol contents were also positively correlated with potassium and calcium contents in leaf $\left(0.553^{* *}\right.$ and $0.606^{* *}$, $0.569^{* *}$ and $\left.0.633^{* * *}\right)$, root $\left(0.555^{* *}\right.$ and $0.675^{* * *}, 0.674^{* *}$ and $\left.0.461^{*}\right)$, and stem $\left(0.470^{*}\right.$ and $0.673^{* * *}$, $0.749^{* * *}$ and $\left.0.437^{*}\right)$, respectively. Furthermore, a positive correlation was also recorded between nitrogen and phosphorus contents with Toc levels in studied plant tissues such as in leaf $\left(0.610^{* *}\right.$ and $0.613^{* *}, 0.539^{* *}$, and $\left.0.683^{* * *}\right)$, $\operatorname{root}\left(0.669^{* * *}, 0.494^{*}\right.$ and $0.488^{*}$ and $0.729^{* * *}, 0.430^{*}$ and $\left.0.620^{* *}\right)$, and stem $\left(0.626^{* * *}\right.$ and $0.601^{* *}, 0.536^{* *}$, and $\left.0.688^{* * *}\right)$, respectively. Figure 3 shows the PCA analysis of varying studied attributes that confirmed correlation studies. Of the extracted components, F1 has a major contribution $(67.43 \%)$ that has divided the studied attributes in different groups. Of them, the major group encircled has parameters that are positively correlated include Pr L, RFW, RDW, N R, S L, SDW, K L, Ca L, P S, P L, K S, and LRWC, and L A, Ca R, Ca S, Mg L, Pr R, P R, N R, and N L contributed maximally in determining the variance. The F2 component has less variance $(17.70 \%)$. Both components have a total variance of $80.13 \%$ (Figure 3; Table 6).

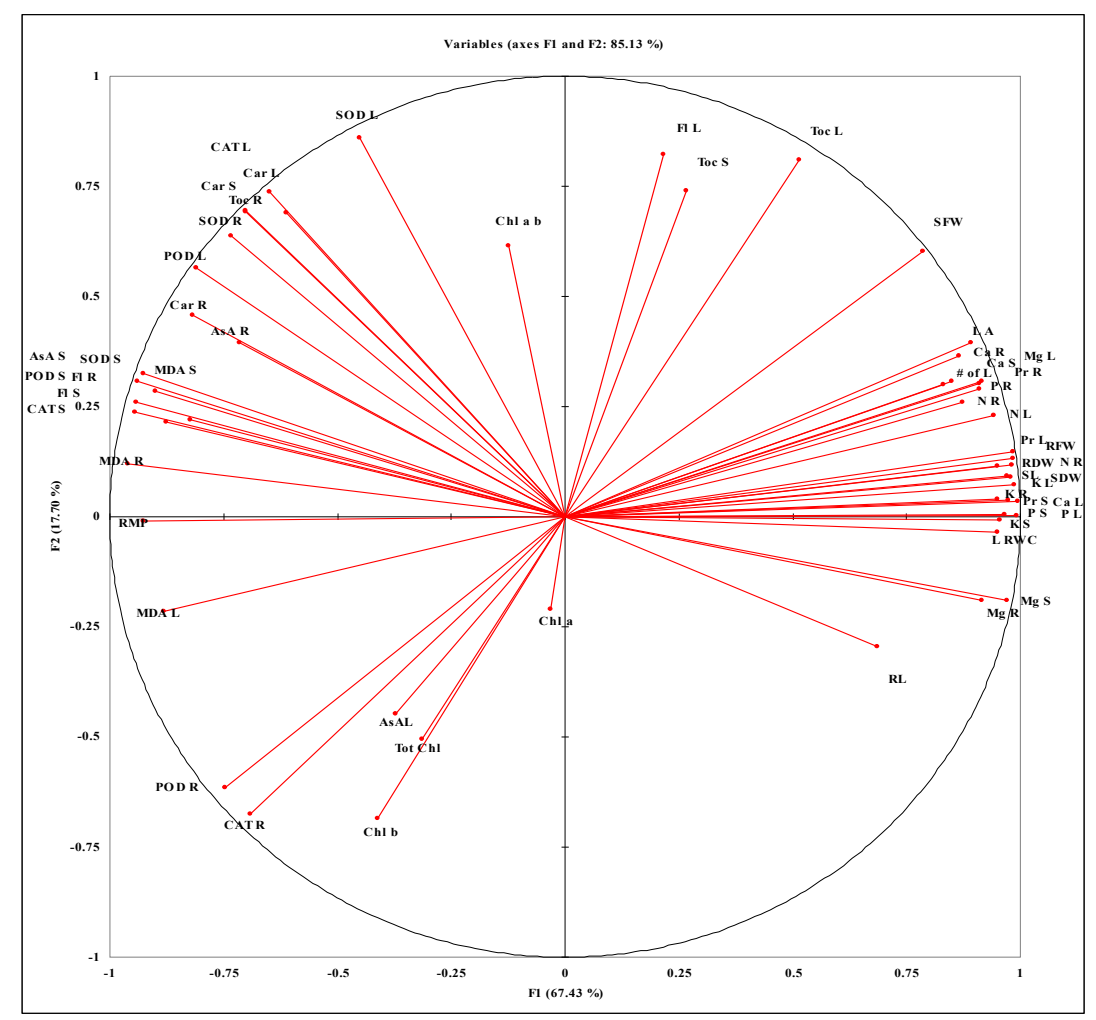

Figure 3. Principle component analysis of tocopherol levels in different plant tissues of maize with studied growth and physio-biochemical attributes, and nutrient accumulation. 
Table 6. Spearman correlation coefficient values $\left(r^{2}\right)$ of Toc levels in different plant parts of maize with growth, biochemical attributes, and nutrient uptake.

\begin{tabular}{|c|c|c|c|}
\hline & Toc L & Toc $\mathbf{R}$ & Toc $S$ \\
\hline Toc L & 1.000 & & \\
\hline Toc R & $0.204 \mathrm{~ns}$ & 1.00 & \\
\hline Toc $S$ & $0.847^{* * *}$ & $0.302 \mathrm{~ns}$ & 1.00 \\
\hline SL & $0.534^{* *}$ & $-0.644^{* * *}$ & $0.287 \mathrm{~ns}$ \\
\hline RL & $0.192 \mathrm{~ns}$ & $-0.661^{* * *}$ & $0.122 \mathrm{~ns}$ \\
\hline NL & $0.797^{* * *}$ & $-0.353 \mathrm{~ns}$ & $0.557^{* *}$ \\
\hline $\mathrm{L} \mathrm{A}$ & $0.768^{* * *}$ & $-0.371 \mathrm{~ns}$ & $0.664^{* * *}$ \\
\hline SFW & $0.921^{* * *}$ & $-0.124 \mathrm{~ns}$ & $0.661^{* * *}$ \\
\hline SDW & $0.578^{* *}$ & $0.643^{* * *}$ & $0.368 \mathrm{~ns}$ \\
\hline RFW & $0.627^{* * *}$ & $-0.597^{* *}$ & $0.381 \mathrm{~ns}$ \\
\hline RDW & $0.780^{* * *}$ & $-0.420^{*}$ & $0.531 \mathrm{~ns}$ \\
\hline L RWC & $0.391 \mathrm{~ns}$ & $0.721^{* * *}$ & $0.164 \mathrm{~ns}$ \\
\hline Chl. a & $-0.088 \mathrm{~ns}$ & $-0.059 \mathrm{~ns}$ & $0.266 \mathrm{~ns}$ \\
\hline Chl. b & $-0.787^{* * *}$ & $-0.181 \mathrm{~ns}$ & $-0.423^{*}$ \\
\hline Chl. a/b & $0.553 * *$ & $0.538^{* *}$ & $0.740^{* * *}$ \\
\hline Tot Chl. & $-0.624^{* * *}$ & -0.133 ns & $-0.239 \mathrm{~ns}$ \\
\hline RMP & $-0.416^{*}$ & $0.664^{* * *}$ & $-0.084 \mathrm{~ns}$ \\
\hline MDA L & $-0.534^{* *}$ & $-0.463 *$ & $-0.202 \mathrm{~ns}$ \\
\hline MDA R & $-0.342 \mathrm{~ns}$ & $-0.751^{* * *}$ & $-0.052 \mathrm{~ns}$ \\
\hline MDA S & $-0.240 \mathrm{~ns}$ & $-0.845^{* * *}$ & $-0.037 \mathrm{~ns}$ \\
\hline Protien L & $0.561 * *$ & $0.591 * *$ & $0.392 \mathrm{~ns}$ \\
\hline Protein R & $0.695^{* * *}$ & $0.324 \mathrm{~ns}$ & $0.314 \mathrm{~ns}$ \\
\hline Protein S & 0.468 * & $0.666^{* * *}$ & $0.159 \mathrm{~ns}$ \\
\hline AsA L & $-0.434 *$ & $-0.115 \mathrm{~ns}$ & $-0.149 \mathrm{~ns}$ \\
\hline AsA R & $-0.180 \mathrm{~ns}$ & $0.709^{* * *}$ & $-0.023 \mathrm{~ns}$ \\
\hline AsA S & $-0.196 \mathrm{~ns}$ & $0.797^{* * *}$ & $0.145 \mathrm{~ns}$ \\
\hline Car L & $0.191 \mathrm{~ns}$ & $0.925^{* * *}$ & $0.339 \mathrm{~ns}$ \\
\hline Car R & $-0.078 \mathrm{~ns}$ & $0.870^{* * *}$ & $0.190 \mathrm{~ns}$ \\
\hline Car S & $0.218 \mathrm{~ns}$ & $0.974^{* * *}$ & $0.411 \mathrm{~ns}$ \\
\hline Flav L & $0.669^{* * *}$ & $0.443^{*}$ & $0.426^{*}$ \\
\hline Flav R & $-0.348 \mathrm{~ns}$ & $0.679^{* * *}$ & $-0.269 \mathrm{~ns}$ \\
\hline Flav S & $-0.211 \mathrm{~ns}$ & $0.733^{* * *}$ & $0.047 \mathrm{~ns}$ \\
\hline CAT L & $0.275 \mathrm{~ns}$ & $0.966^{* * *}$ & 0.434 * \\
\hline CAT R & $-0.884^{* * *}$ & $0.041 \mathrm{~ns}$ & $-0.757^{* * *}$ \\
\hline CAT S & $-0.283 \mathrm{~ns}$ & $0.863^{* * *}$ & $-0.114 \mathrm{~ns}$ \\
\hline POD L & $0.049 \mathrm{~ns}$ & $0.961^{* * *}$ & $0.255 \mathrm{~ns}$ \\
\hline POD R & $-0.831^{* * *}$ & $0.116 \mathrm{~ns}$ & $-0.633^{* * *}$ \\
\hline POD S & $-0.263 \mathrm{~ns}$ & $0.872^{* * *}$ & $-0.107 \mathrm{~ns}$ \\
\hline SOD L & $0.438 *$ & $0.936^{* * *}$ & $0.430 *$ \\
\hline SOD R & $0.173 \mathrm{~ns}$ & $0.951^{* * *}$ & $0.273 \mathrm{~ns}$ \\
\hline SOD S & $-0.220 \mathrm{~ns}$ & $0.859^{* * *}$ & 0.047 ns \\
\hline K L & $0.553^{* *}$ & $0.606^{* *}$ & $0.282 \mathrm{~ns}$ \\
\hline K R & $0.555^{* *}$ & $0.675^{* * *}$ & $0.333 \mathrm{~ns}$ \\
\hline K S & $0.470 *$ & $0.673^{* * *}$ & $0.319 \mathrm{~ns}$ \\
\hline Ca L & $0.569 * *$ & $0.633^{* * *}$ & $0.339 \mathrm{~ns}$ \\
\hline Ca R & $0.674^{* * *}$ & $0.461 *$ & $0.416^{*}$ \\
\hline $\mathrm{Ca} \mathrm{S}$ & $0.749^{* * *}$ & 0.437 * & $0.469 *$ \\
\hline $\mathrm{Mg} \mathrm{L}$ & $0.656^{* * *}$ & $0.439 *$ & 0.470 * \\
\hline $\mathrm{Mg} \mathrm{R}$ & $0.372 \mathrm{~ns}$ & $0.761^{* * *}$ & $0.201 \mathrm{~ns}$ \\
\hline $\mathrm{MgS}$ & $0.335 \mathrm{~ns}$ & $0.833^{* * *}$ & $0.151 \mathrm{~ns}$ \\
\hline N L & $0.610^{* *}$ & $0.613^{* *}$ & $0.368 \mathrm{~ns}$ \\
\hline N R & $0.669^{* * *}$ & 0.494 * & 0.488 * \\
\hline N R & $0.626^{* * *}$ & $0.601^{* *}$ & $0.337 \mathrm{~ns}$ \\
\hline P L & $0.539 * *$ & $0.683^{* * *}$ & 0.383 ns \\
\hline P R & $0.729 * * *$ & $0.430^{*}$ & $0.620^{* *}$ \\
\hline P S & $0.536^{* *}$ & $0.688^{* * *}$ & $0.281 \mathrm{~ns}$ \\
\hline
\end{tabular}

${ }^{*}, * *$ and ${ }^{* * *}=$ Significant at $0.5,0.1$ and 0.01 levels respectively; ns = non-significant. 


\section{Discussion}

The exogenous application of water-soluble antioxidants have been widely investigated to improve stress tolerance, but plant growth modulations by foliar application of lipophilic antioxidants like $\alpha$-Toc has been little studied, probably due to limited information regarding their application, absorption, and translocation within the plant. Kumar et al. [60] and Ali et al. [61] reported that the exogenously applied $\alpha$-Toc can partly alleviate the deleterious impacts of heat and water stress in wheat. In another study, it was found that the exogenous application of $\alpha$-Toc effectively decreased the adverse effects of salt stress in flax cultivars [44]. In most of the earlier studies, the $\alpha$-Toc was applied at adult growth stages. However, the seedling stage (among other growth stages) is considered important due to its involvement in better seed yield by establishing better crop stand [14]. In view of the available information in literature, the present experiment was planned with the objective to study the involvement of $\alpha$-Toc in the improvement of water stress tolerance in relation to the growth modulations of maize depending upon tissue specific partitioning of macro-nutrients and antioxidants in relation with its own translocation/synthesis in specific terms. For this purpose, the response of selected maize genotypes (Agaiti-2002 and EV-1098) was examined under water stress at an early growth stage with and without foliar spray of $\alpha$-Toc.

\subsection{Tocopherol Content in Different Plant Parts}

Foliar spray of $\alpha$-Toc significantly increased the leaf tocopherol levels under non-stressed and stressed conditions, which pointed out the existence of an appropriate mechanism for the uptake of $\alpha$-Toc in the leaves of maize. The increments in root tocopherol contents exhibited a similar pattern, as did the leaves, after foliar application, which suggests an efficient basipetal translocation of $\alpha$-Toc in maize. Our findings are in agreement with Kumar et al. [60], who reported an elevation in the endogenous levels of $\alpha$-Toc in heat-stressed wheat plants after its exogenous application. Furthermore, it has been reported that the exogenous application of these organic compounds, along with altering the cellular metabolic activities, also controls the plant's own metabolism. In the present study, the improvement in the internal levels of $\alpha$-Toc by its exogenous application might also be due to its involvement in regulating plant metabolism $[34,36,40]$.

\subsection{Growth, Water Relations, and Photosynthetic Pigments}

Seedling growth of maize plants was adversely affected in plants grown without foliar application of $\alpha$-Toc under water stress, which is in line with the findings that drought-caused growth reduction is a clear phenomenon in crop plants $[8,14]$. Similarly, in the present study, a drought-induced decrease was recorded in root and shoot lengths, root and shoot fresh and dry weights, leaf area, and number of leaves of both maize genotypes. Growth is dependent on physiological factors, including the content of plant photosynthetic pigments and water relations that directly influences the leaf photosynthetic rate by affecting the capacity of light capturing and assimilation process $[61,62]$. Different plant species and even cultivars in the same species have different potentials to tolerate the adverse conditions regarding these attributes [63].

In the present study, water-stress-induced reduction in biomass is associated with reduced photosynthetic pigment along with disturbed plant water relations, and this reduction was less in cv. Agaiti-2002, showing its better tolerance to drought [34]. The foliar spray of $\alpha$-Toc substantially elevated the plant's endogenous levels and resulted in significant growth improvement under stressed and non-stressed conditions. Increments in plant biomass production is positively associated with the improvement in plant water relations and biosynthesis of biosynthetic pigments such as chlorophyll and carotenoids under the influence of $\alpha$-Toc foliar application. The increment in plant water status might probably be due to impact of $\alpha$-Toc on H-ATPase system showing its role in cellular osmotic adjustment, due to a necessary part of cellular membranes. This involvement of alpha tocopherol in cellular osmotic adjustment confers its role in maintaining the cellular water relations under 
stressful conditions. Similar might be in present study where foliar application of alpha tocopherol improved the leaf relative water content of water stressed maize plants. This improvement in plant water relations further confers its role in improving the leaf net photosynthetic efficiency because plant better water content is necessary to regulate stomatal regulation for better photosynthesis [62]. Furthermore, it is found that $\alpha$-Toc, being a part of cellular membranes, plays a significant role in decreasing the degradation of photosynthetic pigments in a stressful environment [64]. Tocopherols also protect $\mathrm{D}_{1}$ protein [65] and chloroplastic membranes from damaging effects when grown under stressful conditions.

In the present study, foliar-applied $\alpha$-Toc under drought stress further enhanced its internal levels in parallel with the improvement in leaf photosynthetic pigments, which might be due to the significant role of alpha tocopherol in reducing the adverse effects on leaf photosynthetic pigments, resulting in improved photosynthetic efficiency along with better plant water relations that resulted in better plant biomass production. In an earlier study, it was found by Sakr and El-Metwally [43] and El-Quesni [66] in wheat and Hibiscus rosa sinensis, respectively, that exogenous application of $\alpha$-Toc enhanced plant biomass production, which might be due to the role of $\alpha$-Toc in the accumulation of total carbohydrates and protein biosynthesis, confirming its role in photosynthesis and assimilation [67]; this can be correlated with present findings, where higher biomass production was associated with $\alpha$-Toc levels in different parts that improved plant water relations and net photosynthesis as a result of better net assimilation with improved biomass production. Furthermore, this study reveals the increased plant dry weights due to foliar application of $\alpha$-Toc, which points toward the improved photosynthetic activity and assimilation with the establishment of new binding sites [68] after its exogenous application.

Furthermore, in the present study, both maize cultivars maintained an optimum level of their carotenoid contents even under drought and $\alpha$-Toc supplementation, which further enhanced the plant carotenoid contents, especially in leaf and root. These observations point out that $\alpha$-Toc-induced improvement in the growth of maize plants might be due to an improvement in the contents of accessory pigments as additional support to different photosynthetic attributes. In an earlier study, it was found that, in different wheat cultivars [43] and Vicia faba [69], foliar-applied $\alpha$-Toc improved the leaf carotenoid concentration in association with its enhanced growth. Without $\alpha$-Toc application, a decrease in leaf water contents was found in maize plants, which is a well-known phenomenon in all plants. $\alpha$-Toc foliar application significantly increased the leaf water content of water-stressed maize plants, showing its protective role in drought-stressed plants, which might be due to its role in the management of cellular turgor potential through imparting its role in cellular osmotic adjustment by enhancing biosynthesis of osmolytes [7], resulting in better growth by providing an environment for increased cell division and provide an environment for better photosynthesis.

\subsection{Lipid Peroxidation and Antioxidative Defence Mechanism}

An increase in the levels of ROS under stressful environment is a general phenomenon due to $\mathrm{O}_{2}$ excitation to form singlet oxygen or its conversion to hydroxyl radicals $\left(\mathrm{OH}^{-}\right)$, hydrogen peroxide $\left(\mathrm{H}_{2} \mathrm{O}_{2}\right)$, or superoxide $\left(\mathrm{O}^{-2}\right)$ due to the transfer of excited electrons, respectively [34,70], with restricted $\mathrm{e}^{-}$transfer at different steps in photosynthesis and respiration under reduced metabolic activities. These overly produced ROS directly affect different cellular membranes through lipid peroxidation. As a defense for the protection of the cellular membranes and other components from the deleterious and damaging effects of overproduced ROS, plants have evolved well-developed mechanisms for the antioxidation of ROS, i.e., comprised of non-enzymatic (AsA, phenolics, carotenoids, flavonoids, tocopherol, etc.) and enzymatic (SOD, POD, CAT, APX) components $[14,34,71]$. This antioxidative system works well in combination. In the present study, the $\alpha$-Toc-treated plants suffered significantly lower oxidative damage, especially in root and leaf, as depicted by the lower MDA contents in these plant parts relative to untreated ones (as reported earlier for wheat) [60]. Drought stress significantly increased oxidative stress in maize plants; this is obvious 
from increased levels of MDA, a product of lipid peroxidation. Damage to biological membranes due to oxidative stress is a general phenomenon that generally increases in specific environments $[14,45]$. In an earlier study, significantly lower oxidative stress was recorded in $\alpha$-Toc applied plants as obvious from lower membrane permeability which is in line with its role in quenching lipid peroxyl radicals, responsible for propagating lipid peroxidation $[69,72,73]$. It was reported that during early growth stages, $\alpha$-Toc played a significant role in counteracting the adverse effects of membrane lipid peroxidation. Furthermore, being lipophilic, $\alpha$-Toc has a significant role in membrane stabilization [74] and also protects them from ROS [75]. Furthermore, $\alpha$-Toc directly scavenges singlet oxygen [76], giving rise an intermediate tocopherol quinone, which again yields $\alpha$-Toc in chloroplasts, thereby conferring the recycling for oxidized tocopherols [77]. Reports exist that $\alpha$-Tocopherol is also an excellent quencher and scavenger of singlet oxygen by controlling the lifetime of ROS. By resonance energy transfer, one $\alpha$-Toc molecule can neutralize up to 120 molecules of singlet oxygen [78]. The activities of antioxidants such as SOD, POD, and CAT were found to be higher in leaves and roots of maize plant after $\alpha$-Toc treatment, which suggested their antioxidative role to be stimulated in the presence of $\alpha$-Toc.

Higher activity rates of these enzymes were found in leaves and roots where more accumulation of $\alpha$-Toc was found in comparison with stem, showing the supportive role of $\alpha$-Toc in the activities of antioxidative enzymes. Furthermore, the higher levels of non-enzymatic antioxidant in root and leaf as compared to stem (such as AsA, phenolics, and flavonoids) are also associated with high content of $\alpha$-Toc in these plant parts. These findings show that $\alpha$-Toc application after its translocation to the studied plant parts played a significant role in increasing the activities of antioxidative enzymes and the levels of non-enzymatic antioxidant compounds and thus played an imperative role in protecting cellular membranes by boosting the plant's own mechanism. It was found by Fahrenholtz et al. [79] that $\alpha$-Toc acts as an antioxidative defense mechanism in plants. It was also found that $\alpha$-Toc minimizes the oxidative changes in the cellular membrane in a significant way with other antioxidants [80-82].

\subsection{Uptake of Mineral Nutrients}

Drought-induced growth reduction can also be attributed to disturbances in the uptake of mineral nutrients along with other physiological attributes. It is well known that disturbance or reductions in the leaf uptake of mineral nutrient in plants is probably due to nutrient availability, partitioning, and transport, which is negatively affected under drought conditions. Plant mineral nutrients status played a major role in determining drought tolerance [83]. In the present study, the PCA analysis and the correlations studied suggest that an improvement in the levels of $\alpha$-Toc contents in different plant parts induced by its foliar application increased the uptake of mineral nutrients $(\mathrm{K}, \mathrm{Ca}, \mathrm{N}$, and $\mathrm{P})$. Mineral nutrients effectively decrease the harsh effects of water stress by various mechanisms [22]. For example, it has been found that better uptake of mineral nutrients like $\mathrm{Ca}^{2+}$, $\mathrm{N}$, and $\mathrm{K}^{+}$reduces the deleterious effects of over produced ROS by increasing the concentration of antioxidants like CAT, POD, and SOD [22]. It has been reported that $\mathrm{P}, \mathrm{K}$, and $\mathrm{Mg}$ improve root growth, which results in improved water intake conferring the drought tolerance. It can be interpreted that optimum nutrient levels maintained after $\alpha$-Toc application confer drought tolerance induction in maize plants in parallel with improved growth. This is more likely because leaf water contents were significantly improved by foliar spray of $\alpha$-Toc. The supportive role of $\alpha$-Toc after its application in the absorption of nutrients from the soil in stressful environment has been found extensively $[44,78]$, and it is reported that $\alpha$-Toc induced increase uptake of nutrients due to $\alpha$-Toc being an antioxidant, along with membrane permeability. Furthermore, previous studies found that $\alpha$-Toc induced an increase in growth, water relation, and nutrient uptake associated with improved stem and leaf anatomy, which further improved translocation to different plant parts. Therefore, the studies confirm that, as in the present study, $\alpha$-Toc application might improve the uptake and translocation of different nutrients from the soil solution to the roots and then to different plant parts, resulting in better assimilation and growth. 


\section{Conclusions}

It can be concluded that endogenous levels of $\alpha$-Toc have an important role in enhancing water stress tolerance of maize cultivars, and its foliar application is found to be effective in reducing water-stress-induced adversative effects on growth by modulating different metabolic activities. Our results confirmed that $\alpha$-Tocopherol application resulted in membrane protection through increased activities of antioxidative enzymes (CAT, POD, and SOD) and the content of non-enzymatic antioxidants with improved water relations. The correlations and PCA analysis revealed that the increase in $\alpha$-Tocopherol contents in different plant parts after its foliar application increased the uptake of mineral nutrients $\left(\mathrm{K}^{+}, \mathrm{Ca}^{2+}, \mathrm{N}\right.$, and $\left.\mathrm{P}\right)$. Optimum water content and nutrients, along with better antioxidant potential, ultimately resulted in drought tolerance in both maize cultivars that increased growth. In relation to translocation-dependent effects, it was found that $\alpha$-Toc followed basipetal translocation, concentrating mainly in the roots rather than the shoot after its foliar application. Therefore, analysis of the impact of foliar application of $\alpha$-Toc on seed yield and nutritional quality of arable crops under stressful environment should be the subject of future studies.

Author Contributions: Conceptualization, Q.A., M.R., S.A., M.N.A., H.A.E.-S., and F.A.A.-M.; Data curation, Q.A., M.T.J., M.Z.H., and R.P.; Formal analysis, Q.A., M.T.J., N.H., and R.P.; Funding acquisition, Q.A., M.N.A., H.A.E.-S., and F.A.A.-M.; Investigation, N.H. and R.P.; Methodology, M.T.J., M.Z.H., and R.P.; Resources, M.R., S.A., M.N.A., H.A.E.-S., and F.A.A.-M.; Software, M.R., S.A., M.N.A., H.A.E.-S., and F.A.A.-M.; Supervision, Q.A. and M.T.J.; Validation, M.T.J., M.Z.H., and N.H.; Visualization, M.Z.H. and N.H.; Writing—original draft, Q.A. and F.A.A.-M.; Writing-review and editing, M.R., S.A., M.N.A., and H.A.E.-S. All authors have read and agreed to the published version of the manuscript.

Funding: The authors are grateful to the Government College University, Faisalabad, Pakistan for its support. The authors would like to extend their sincere appreciation to the Researchers Supporting Project Number (RSP-2020/19), King Saud University, Riyadh, Saudi Arabia.

Acknowledgments: The authors are grateful to the Higher Education Commission (HEC) Islamabad, Pakistan for its support. The authors would like to extend their sincere appreciation to the Researchers Supporting Project Number (RSP-2020/19), King Saud University, Riyadh, Saudi Arabia.

Conflicts of Interest: The authors declare that there are no conflicts of interest regarding the publication of this paper.

\section{Abbreviations}

$\begin{array}{ll}\text { Toc L } & \text { leaf tocopherol } \\ \text { Toc R } & \text { root tocopherol } \\ \text { Toc S } & \text { stem tocopherol } \\ \text { SL } & \text { shoot length } \\ \text { RL } & \text { root length } \\ \text { NL } & \text { number of leaves } \\ \text { LA } & \text { leaf area } \\ \text { SFW } & \text { shoot fresh weight } \\ \text { SDW } & \text { shoot dry weight } \\ \text { RFW } & \text { root fresh weight } \\ \text { RDW } & \text { root dry weight } \\ \text { L RWC } & \text { leaf relative water content } \\ \text { Chl. a } & \text { chlorophyll a } \\ \text { Chl. b } & \text { chlorophyll b } \\ \text { Chl. a/b } & \text { chlorophyll a/b ratio } \\ \text { Tot Chl. } & \text { total chlorophyll } \\ \text { RMP } & \text { relative membrane permeability } \\ \text { MDA L } & \text { MDA leaf } \\ \text { MDA R } & \text { MDA root } \\ \text { MDA S } & \text { MDA stem } \\ \text { Protien L } & \text { protein leaf } \\ \text { Protein R } & \text { protein root } \\ \text { Protein S } & \text { protein stem }\end{array}$




$\begin{array}{ll}\text { AsAL } & \text { ascorbic acid leaf } \\ \text { AsA R } & \text { ascorbic acid root } \\ \text { AsA S } & \text { ascorbic acid stem } \\ \text { Car L } & \text { carotenoids leaf } \\ \text { Car R } & \text { carotenoids root } \\ \text { Car S } & \text { carotenoids stem } \\ \text { Flav L } & \text { flavonoids leaf } \\ \text { Flav R } & \text { flavonoids root } \\ \text { Flav S } & \text { flavonoids stem } \\ \text { CAT L } & \text { catalase leaf } \\ \text { CAT R } & \text { catalase root } \\ \text { CAT S } & \text { catalase stem } \\ \text { POD L } & \text { peroxidase leaf } \\ \text { POD R } & \text { peroxidase root } \\ \text { POD S } & \text { peroxidase stem } \\ \text { SOD L } & \text { superoxide dismutase leaf } \\ \text { SOD R } & \text { superoxide dismutase root } \\ \text { SOD S } & \text { superoxide dismutase stem } \\ \text { K L } & \text { potassium leaf } \\ \text { K R } & \text { potassium root } \\ \text { K S } & \text { potassium stem } \\ \text { Ca L } & \text { calcium leaf } \\ \text { Ca R } & \text { calcium root } \\ \text { Ca S } & \text { calcium stem } \\ \text { Mg L } & \text { magnesium leaf } \\ \text { MgR } & \text { magnesium root } \\ \text { Mg S } & \text { magnesium stem } \\ \text { N L } & \text { nitrogen leaf } \\ \text { N R } & \text { nitrogen root } \\ \text { N S } & \text { nitrogen stem } \\ \text { P L } & \text { phosphorus leaf } \\ \text { P R } & \text { phosphorus root } \\ \text { P S } & \text { phosphorus stem } \\ & \end{array}$

\section{References}

1. Gobin, A. Impact of Heat and Drought Stress on Arable Crop Production in Belgium. Nat. Hazards Earth Syst. Sci. 2012, 12, 1911-1922. [CrossRef]

2. Khan, N.; Bano, A. Rhizobacteria and Abiotic Stress Management. In Plant Growth Promoting Rhizobacteria for Sustainable Stress Management; Springer: Singapore, 2019; pp. 65-80.

3. Cairns, J.E.; Sanchez, C.; Vargas, M.; Ordoñez, R.; Araus, J.L. Dissecting Maize Productivity: Ideotypes Associated with Grain Yield Under Drought Stress and Well-Watered Conditions. J. Integr. Plant Biol. 2012, 54, 1007-1020. [CrossRef] [PubMed]

4. Adnan, S.; Ullah, K.; Gao, S.; Khosa, A.H.; Wang, Z. Shifting of Agro Climatic Zones, their Drought Vulnerability, and Precipitation and Temperature Trends in Pakistan. Int. J. Climatol. 2017, 37, 529-543. [CrossRef]

5. Kazmi, D.H.; Li, J.; Rasul, G.; Tong, J.; Ali, G.; Cheema, S.B.; Liu, L.; Gemmer, M.; Fisherr, T. Statistical Downscaling and Future Scenario Generation of Temperatures for Pakistan Region. Theor. Appl. Climatol. 2015, 120, 341-350. [CrossRef]

6. Hina, S.; Saleem, F. Historical Analysis (1981-2017) of Drought Severity and Magnitude over a Predominantly Arid Region of Pakistan. Clim. Res. 2019, 78, 189-204. [CrossRef]

7. Um, M.; Kim, Y.; Park, D.; Jung, K.; Wang, Z.; Kim, M.M.; Shin, H. Impacts of Potential Evapotranspiration on Drought Phenomena in Different Regions and Climate Zones. Sci. Total Environ. 2020, 703, 135590. [CrossRef]

8. Ali, Q.; Anwar, F.; Ashraf, M.; Saari, N.; Perveen, R. Ameliorating Effects of Exogenously Applied Proline on Seed Composition, Seed Oil Quality and Oil Antioxidant Activity of Maize (Zea mays L.) Under Drought Stress. Int. J. Mol. Sci. 2013, 14, 818-835. [CrossRef] 
9. Khan, N.; Bano, A.; Rahman, M.A.; Guo, J.; Kang, Z.; Babar, M.A. Comparative physiological and metabolic analysis reveals a complex mechanism involved in drought tolerance in chickpea (Cicer arietinum L.) induced by PGPR and PGRs. Sci. Rep. 2019, 9, 1-19. [CrossRef]

10. Rana, V.; Singh, D.; Dhiman, R.; Chaudhary, H.K. Evaluation of Drought Tolerance Among Elite Indian Bread Wheat Cultivars. Cereal. Res. Commun. 2014, 42, 91-101. [CrossRef]

11. Chaves, M.M.; Maroco, J.P.; Pereira, J.S. Understanding Plant Responses to Drought From Genes to the Whole Plant. Physiol. Mol. Biol. Plants 2003, 30, 239-264. [CrossRef]

12. Khan, N.; Bano, A.; Rahman, M.A.; Rathinasabapathi, B.; Babar, M.A. UPLC-HRMS-based untargeted metabolic profiling reveals changes in chickpea (Cicer arietinum) metabolome following long-term drought stress. Plant Cell Environ. 2019, 42, 115-132. [CrossRef] [PubMed]

13. Demirevska, K.; Simova-Stoilova, L.; Fedina, I.; Georgieva, K.; Kunert, K. Response of Oryza cystatin L. Transformed Tobacco Plants to Drought, Heat and Light Stress. J. Agron. Crop Sci. 2010, 196, 90-99. [CrossRef]

14. Ali, Q.; Javed, M.T.; Noman, A.; Haider, M.Z.; Waseem, M.; Iqbal, N.; Perveen, R. Assessment of Drought Tolerance in Mung Bean Cultivars/Lines as Depicted by the Activities of Germination Enzymes, Seedling's Antioxidative Potential and Nutrient Acquisition. Arch. Agron. Soil. Sci. 2018, 64, 84-102. [CrossRef]

15. Chen, J.; Junying, D. Effect of Drought on Photosynthesis and Grain Yield of Corn Hybrids With Different Drought Tolerance. Acta-Agron Sin. 1996, 22, 757-762.

16. Khan, N.; Bano, A.; Curá, J.A. Role of Beneficial Microorganisms and Salicylic Acid in Improving Rainfed Agriculture and Future Food Safety. Microorganisms 2020, 8, 1018. [CrossRef]

17. Lawlor, D.W.; Cornic, G. Photosynthetic Carbon Assimilation and Associated Metabolism in Relation to Water Deficits in Higher Plants. Plant Cell Environ. 2002, 25, 275-294. [CrossRef]

18. Rapacz, M.; Kościelniak, J.; Jurczyk, B.; Adamska, A.; Wójcik, M. Different Patterns of Physiological and Molecular Response to Drought in Seedlings of Malt- and Feed-Type Barleys (Hordeum vulgare). J. Agron. Crop Sci. 2010, 196, 9-19. [CrossRef]

19. Marschner, P. Mineral Nutrition of Higher Plants; Academic Press: London, UK, 2011.

20. Mukhtar, I.; Shahid, M.A.; Khan, M.W.; Balal, R.M.; Iqbal, M.M.; Naz, T.; Zubair, M.; Ali, H.H. Improving salinity tolerance in chili by exogenous application of calcium and sulphur. Soil Environ. 2016, 1, 35.

21. Heidari, M.; Karami, V. Effects of Different Mycorrhiza Species on Grain Yield, Nutrient Uptake and Oil Content of Sunflower Under Water Stress. J. Saudi Soc. Agric. Sci. 2014, 13, 9-13. [CrossRef]

22. Waraich, E.A.; Ahmad, R.; Ashraf, M.Y.; Ahmad, S.M. Improving Agricultural Water Use Efficiency by Nutrient Management in Crop Plants. Acta Agric. Scand. B Soil Plant Sci. 2011, 61, 291-304. [CrossRef]

23. Fahad, S.; Bajwa, A.A.; Nazir, U.; Anjum, S.A.; Farooq, A.; Zohaib, A.; Ihsan, M.Z. Crop Production Under Drought and Heat Stress: Plant Responses and Management Options. Front. Plant Sci. 2017, 8, 1147. [CrossRef] [PubMed]

24. Hussain, H.A.; Hussain, S.; Khaliq, A.; Ashraf, U.; Anjum, S.A.; Men, S.; Wang, L. Chilling and Drought Stresses in Crop Plants: Implications, Cross Talk, and Potential Management Opportunities. Front. Plant Sci. 2018, 9, 393. [CrossRef]

25. Rajala, A.; Niskanen, M.; Isolahti, M.; Peltonen-Sainio, P. Seed Quality Effects on Seedling Emergence, Plant Stand Establishment and Grain Yield in Two-Row Barley. Agric. Food Sci. 2011, 20, 228-234. [CrossRef]

26. Singla, S.; Grover, K.; Angadi, S.V.; Schutte, B.; VanLeeuwen, D. Guar Stand Establishment, Physiology, and Yield Responses to Planting Date in Southern New Mexico. Agron. J. 2016, 8, 2289-2300. [CrossRef]

27. Srivalli, B.; Sharma, G.; Khanna-Chopra, R. Antioxidative Defense System in an Upland Rice Cultivar Subjected to Increasing Intensity of Water Stress Followed by Recovery. Physiol. Plant. 2003, 119, 503-512. [CrossRef]

28. Ashraf, M. Inducing Drought Tolerance in Plants: Recent Advances. Biotechnol. Adv. 2010, 28, 169-183. [CrossRef]

29. Zhang, L.X.; Li, S.X.; Zhang, H.; Liang, Z.S. Nitrogen Rates and Water Stress Effects on Production, Lipid Peroxidation and Antioxidative Enzyme Activities in Two Maize (Zea mays L.) genotypes. J. Agron. Crop Sci. 2007, 193, 387-397. [CrossRef]

30. Farooq, M.; Wahid, A.; Lee, D.J.; Cheema, S.A.; Aziz, T. Comparative Time Course Action of the Foliar Applied Glycinebetaine, Salicylic Acid, Nitrous Oxide, Brassinosteroids and Spermine in Improving Drought Resistance of Rice. J. Agron. Crop Sci. 2010, 196, 336-345. [CrossRef] 
31. Posmyk, M.M.; Kontek, R.; Janas, K.M. Antioxidant Enzymes Activity and Phenolic Compounds Content in Red Cabbage Seedlings Exposed to Copper Stress. Ecotoxicol. Environ. Saf. 2009, 72, 596-602. [CrossRef]

32. Farooq, M.; Basra, S.M.A.; Wahid, A.; Cheema, Z.A.; Cheema, M.A.; Khaliq, A. Physiological Role of Exogenously Applied Glycinebetaine to Improve Drought Tolerance in Fine Grain Aromatic Rice (Oryza sativa L.). J. Agron. Crop. Sci. 2008, 194, 325-333. [CrossRef]

33. Khan, S.; Hasan, M.; Bari, A.; Khan, F. Climate Classification of Pakistan. In Proceedings of the Balwois Conference, Ohrid, Macedonia, 25-29 May 2010; pp. 1-47.

34. Ali, Q.; Ashraf, M. Induction of Drought Tolerance in Maize (Zea mays L.) Due to Exogenous Application of Trehalose: Growth, Photosynthesis, Water Relations and Oxidative Defence Mechanism. J. Agron. Crop Sci. 2011, 197, 258-271. [CrossRef]

35. Tayebi-Meigooni, A.; Awang, Y.; Biggs, A.R.; Mohamad, R.; Madani, B.; Ghasemzadeh, A. Mitigation of Salt-Induced Oxidative Damage in Chinese Kale (Brassica alboglabra L.) Using Ascorbic Acid. Acta Agric. Scand. Sect. B-Soil Plant Sci. 2014, 64, 13-23.

36. Noman, A.; Ali, Q.; Maqsood, J.; Iqbal, N.; Javed, M.T.; Rasool, N.; Naseem, J. Deciphering Physio-Biochemical, Yield, and Nutritional Quality Attributes of Water-Stressed Radish (Raphanus sativus L.) Plants Grown From Zn-Lys Primed Seeds. Chemosphere 2018, 195, 175-189. [CrossRef] [PubMed]

37. Khan, N.; Bano, A.; Zandi, P. Effects of exogenously applied plant growth regulators in combination with PGPR on the physiology and root growth of chickpea (Cicer arietinum) and their role in drought tolerance. J. Plant Interact. 2018, 1, 239-247. [CrossRef]

38. Khan, N.; Zandi, P.; Ali, S.; Mehmood, A.; Adnan Shahid, M.; Yang, J. Impact of salicylic acid and PGPR on the drought tolerance and phytoremediation potential of Helianthus annus. Front. Microbiol. 2018, 9, 2507. [CrossRef]

39. Khan, N.; Bano, A.; Ali, S.; Babar, M.A. Crosstalk amongst phytohormones from planta and PGPR under biotic and abiotic stresses. Plant Growth Regul. 2020, 90, 189-203. [CrossRef]

40. Jamil, S.; Ali, Q.; Iqbal, M.; Javed, M.T.; Iftikhar, W.; Shahzad, F.; Perveen, R. Modulations in Plant Water Relations and Tissue-Specific Osmoregulation by Foliar-Applied Ascorbic Acid and the Induction of Salt Tolerance in Maize Plants. Braz. J. Bot. 2015, 38, 527-538. [CrossRef]

41. Hasegawa, P.M.; Bressan, R.A.; Zhu, J.K.; Bohnert, H.J. Plant Cellular and Molecular Responses to High Salinity. Annu. Rev. Plant Biol. 2000, 51, 463-499. [CrossRef]

42. Fritsche, S.; Wang, X.; Jung, C. Recent Advances in Our Understanding of Tocopherol Biosynthesis in Plants: An Overview of Key Genes, Functions, and Breeding of Vitamin E Improved Crops. Antioxidants 2017, 6, 99. [CrossRef]

43. Sakr, M.T.; El-Metwally, M.A. Alleviation of the Harmful Effects of Soil Salt Stress on Growth, Yield and Endogenous Antioxidant Content of Wheat Plant by Application of Antioxidants. Pak. J. Biol. Sci. 2009, 12, 624-630. [CrossRef]

44. Sadak, S.M.; Dawood, S. Role of Ascorbic Acid and $\alpha$ Tocopherol in Alleviating Salinity Stress on Flax Plant (Linum usitatissimum L.). J. Stress Physiol. Biochem. 2014, 10, 93-111.

45. Gill, S.S.; Tuteja, N. Reactive Oxygen Species and Antioxidant Machinery in Abiotic Stress Tolerance in Crop Plants. Plant Physiol. Biochem. 2010, 48, 909-930. [CrossRef]

46. Saleem, A.; Saleem, U.; Subhani, G.M. Correlation and Path Coefficient Analysis in Maize (Zea mays L.). J. Agric. Sci. 2007, 45, 177-183.

47. Aslam, M.; Zamir, M.S.I.; Anjum, S.A.; Khan, I.; Tanveer, M. An Investigation into Morphological and Physiological Approaches to Screen Maize (Zea mays L.) Hybrids for Drought Tolerance. Cereal Res. Commun. 2015, 43, 41-51. [CrossRef]

48. Anjum, S.A.; Ashraf, U.; Tanveer, M.; Khan, I.; Hussain, S.; Shahzad, B.; Zohaib, A.; Abbas, F.; Saleem, M.F.; Ali, I.; et al. Drought Induced Changes in Growth, Osmolyte Accumulation and Antioxidant Metabolism of Three Maize Hybrids. Front. Plant Sci. 2017, 8, 69. [CrossRef]

49. Arnon, D.I. Copper Enzyme in Isolated Chloroplast Polyphenol Oxidase in Beta vulgaris. Plant Physiol. 1949, 24, 1-15.

50. Kirk, J.T.O.; Allen, R.L. Dependence of Chloroplast Pigment Synthesis on Protein Synthesis: Effect of Actidione. Biochem. Biophys. Res. Commun. 1965, 21, 523-530. [CrossRef] 
51. Yang, G.; Rhodes, D.; Joly, R.J. Effects of High Temperature on Membrane Stability and Chlorophyll Fluorescence in Glycinebetaine-Deficient and Glycinebetaine-Containing Maize Lines. Physiol. Mol. Biol. Plants 1996, 23, 437-443. [CrossRef]

52. Cakmak, I.; Horst, W.J. Effect of Aluminium on Lipid Peroxidation, Superoxide Dismutase, Catalase, and Peroxidase Activities in Root Tips of Soybean (Glycine max). Physiol. Plant. 1991, 83, 463-468. [CrossRef]

53. Bradford, M.M.A. Rapid and Sensitive Method for the Quantitation of Microgram Quantities of Protein Utilizing the Principle of Protein-Dye Binding. Anal. Biochem. 1976, 72, 248-254. [CrossRef]

54. Giannopolitis, C.N.; Ries, S.K. Superoxide Occurrence in Higher Plants. Plant Physiol. 1977, 59, $309-314$. [CrossRef] [PubMed]

55. Chance, B.; Maehly, A.C. Assay of Catalase and Peroxidase. Methods Enzymol. 1955, 2, 764-775.

56. Mukherjee, S.P.; Choudhuri, M.A. Implications of Water Stress-Induced Changes in the Levels of Endogenous Ascorbic Acid and Hydrogen Peroxide in Vigna Seedlings. Physiol. Plant. 1983, 58, 166-170. [CrossRef]

57. Karadeniz, F.; Burdurlu, H.S.; Koca, N.; Soyer, Y. Antioxidant Activity of Selected Fruits and Vegetables Grown in Turkey. Turk. J. Agric. For. 2005, 29, 297-303.

58. Backer, H.; Frank, O.; De Angelis, B.; Feingold, S. Plasma Tocopherol in Man at Various Times After Ingesting Free or Acetylated Tocopherol. Nutr. Rep. Int. 1980, 21, 531-536.

59. Bremner, J.M.; Keeney, D.R. Steam Distillation Methods for Determination of Ammonium, Nitrate and Nitrite. Anal. Chim. Acta 1965, 32, 485-495. [CrossRef]

60. Kumar, S.; Singh, R.; Nayyar, H. $\alpha$-Tocopherol Application Modulates the Response of Wheat (Triticum aestivum L.) Seedlings to Elevated Temperatures by Mitigation of Stress Injury and Enhancement of Antioxidants. J. Plant Growth Regul. 2012, 32, 307-314. [CrossRef]

61. Ali, Q.; Ali, S.; Iqbal, N.; Javed, M.T.; Rizwan, M.; Khaliq, R.; Wijaya, L. Alpha-Tocopherol Fertigation Confers Growth Physio-Biochemical and Qualitative Yield Enhancement in Field Grown Water Deficit Wheat (Triticum aestivum L.). Sci. Rep. 2019, 9, 1-15. [CrossRef]

62. Taize, L.; Zeiger, E.; Moller, I.M.; Murphy, A. Plant Physiology and Development, 6th ed.; Sinauer Associates, Inc.: Sunderland, MA, USA, 2015.

63. Foryer, C.; Noctor, G. Oxygen Processing in Photosynthesis: Regulation and Signaling. New Phytol. 2000, 146, 359-388. [CrossRef]

64. Munné-Bosch, S.; Alegre, L. The Function of Tocopherols and Tocotrienols in Plants. Crit. Rev. Plant Sci. 2002, 21, 31-57. [CrossRef]

65. Trebst, A.; Depka, B.; Holländer-Czytko, H. A Specific Role for Tocopherol and of Chemical Singlet Oxygen Quenchers in the Maintenance of Photosystem II Structure and Function in Chlamydomonas reinhardtii. FEBS Lett. 2002, 516, 156-160. [CrossRef]

66. El-Quesni, F.E.M.; Abd El-Aziz, N.G.; Kandil, M.M. Some Studies on the Effect of Ascorbic Acid and a-Tocopherol on the Growth and Some Chemical Composition of Hibiscus rosa sinensis L. at Nubaria. Ozean J. Appl. Sci. 2009, 2, 159-167.

67. Sadak, M.S.; Rady, M.M.; Badr, N.M.; Gaballah, M.S. Increasing Sun Flower Salt Tolerance Using Nicotinamide and a-Tocopherol. Int. J. Acad. Res. 2010, 2, 263-270.

68. Javed, M.T.; Greger, M. Cadmium Triggers Elodea canadensis to Change the Surrounding Water $\mathrm{pH}$ and Thereby Cd uptake. Int. J. Phytoremediation 2010, 13, 95-106. [CrossRef] [PubMed]

69. Orabi, S.A.; Abdelhamid, M.T. Protective Role of $\alpha$-Tocopherol on Two Vicia faba Cultivars Against Seawater-Induced Lipid Peroxidation by Enhancing Capacity of Anti-Oxidative System. J. Saudi Soc. Agric. Sci. 2016, 15, 145-154. [CrossRef]

70. Shigeoka, S.; Ishikawa, T.; Tamoi, M.; Miyagawa, Y.; Takeda, T.; Yabuta, Y.; Yoshimura, K. Regulation and Function of Ascorbate Peroxidase Isoenzymes. J. Exp. Bot. 2002, 53, 1305-1319. [CrossRef]

71. Ali, Q.; Haider, M.Z.; Iftikhar, W.; Jamil, S.; Javed, M.T.; Noman, A.; Perveen, R. Drought Tolerance Potential of Vigna mungo L. Lines as Deciphered by Modulated Growth, Antioxidant Defense, and Nutrient Acquisition Patterns. Braz. J. Bot. 2016, 39, 801-812. [CrossRef]

72. Maeda, H.; Sakuragi, Y.; Bryant, D.A.; DellaPenna, D. Tocopherols Protect Synechocystis sp. Strain PCC 6803 From Lipid Peroxidation. Plant Physiol. 2005, 138, 1422-1435. [CrossRef]

73. Sattler, S.E.; Cahoon, E.B.; Coughlan, S.J.; DellaPenna, D. Characterization of Tocopherol Cyclases From Higher Plants and Cyanobacteria. Evolutionary Implications for Tocopherol Synthesis and Function. Plant Physiol. 2003, 132, 2184-2195. [CrossRef] 
74. Wang, X.; Quinn, P.J. Preferential Interaction of $\alpha$-Tocopherol With Phosphatidylcholines in Mixed Aqueous Dispersions of Phosphatidylcholine and Phosphatidylethanolamine. Eur. J. Biochem. 2000, 267, 6362-6368. [CrossRef]

75. Maeda, H.; DellaPenna, D. Tocopherol Functions in Photosynthetic Organisms. Curr. Opin. Plant Biol. 2007, 10, 260-265. [CrossRef]

76. Fukuzawa, K.; Matsuura, K.; Tokumura, A.; Suzuki, A.; Terao, J. Kinetics and Dynamics of Singlet Oxygen Scavenging by $\alpha$-Tocopherol in Phospholipid Model Membranes. Free Radic. Biol. Med. 1997, 22, 923-930. [CrossRef]

77. Kobayashi, N.; DellaPenna, D. Tocopherol Metabolism, Oxidation and Recycling Under High Light Stress in Arabidopsis. Plant J. 2008, 55, 607-618. [CrossRef]

78. Fahrenholtz, S.R.; Doleiden, F.H.; Trozzolo, A.M.; Lamola, A.A. On the Quenching of Singlet Oxygen by $\alpha$-Tocopherol. J. Photochem. Photobiol. Biol. 1974, 20, 505-509. [CrossRef]

79. Semida, W.M.; Taha, R.S.; Abdelhamid, M.T.; Rady, M.M. Foliar-Applied $\alpha$-Tocopherol Enhances Salt-Tolerance in Vicia faba L. Plants Grown Under Saline Conditions. S. Afr. J. Bot. 2014, 95, $24-31$. [CrossRef]

80. Khattab, H. Role of Glutathione and Polyadenylic Acid on the Oxidative Defense Systems of Two Different Cultivars of Canola Seedlings Grown Under Saline Conditions. Aust. J. Basic Appl. Sci. 2007, 1, 323-334.

81. Pourcel, L.; Routaboul, J.M.; Cheynier, V.; Lepiniec, L.; Debeaujon, I. Flavonoid Oxidation in Plants: From Biochemical Properties to Physiological Functions. Trends Plant Sci. 2007, 12, 29-36. [CrossRef]

82. Shao, H.B.; Chu, L.Y.; Shao, M.A.; Jaleel, C.A.; Hong-mei, M. Higher Plant Antioxidants and Redox Signaling Under Environmental Stresses. C. R. Biol. 2008, 331, 433-441. [CrossRef]

83. Hu, Y.; Schmidhalter, U. Drought and Salinity: A Comparison of Their Effects on Mineral Nutrition of Plants. J. Plant Nutr. Soil Sci. 2005, 168, 541-549. [CrossRef]

(C) 2020 by the authors. Licensee MDPI, Basel, Switzerland. This article is an open access article distributed under the terms and conditions of the Creative Commons Attribution (CC BY) license (http://creativecommons.org/licenses/by/4.0/). 